\title{
Robust Output-Feedback MPC With Integral Action
}

\author{
Christian Løvaas, María M. Seron, and Graham C. Goodwin, Fellow, IEEE
}

\begin{abstract}
In this paper, we propose a robust output-feedback model predictive control (MPC) design for a class of open-loop stable systems, having non-vanishing output disturbances, hard constraints and linear-time invariant model uncertainty. Integral action is included in the proposed design by utilizing a linear estimator for both the output disturbance and the system state. The main distinction from previous work on robust MPC design (with integral action) is that we establish robust convergence in the face of both imperfect state information and dynamic model uncertainty.
\end{abstract}

Index Terms-Model predictive control (MPC), robust control.

\section{INTRODUCTION}

A large portion of the literature on model predictive control (MPC) has been aimed at developing, so called, robust MPC policies. These utilize a description of the model uncertainty and are aimed at guaranteeing both constraint satisfaction and closed-loop stability. For example, the latter two design objectives have been achieved by many authors, subject to the following two assumptions (see, e.g., [1]-[6]): (i) a state-space model with an associated parametric uncertainty description is available (e.g., multi-model or polytopic); and (ii) the state of the actual system is measured at each time step. Note, however, that both these assumptions are difficult to meet, since, in practice, one usually only has input-output data.

There has also been some recent work that aims to address the, arguably more realistic, setup of linear robust control, in which the (constrained) open-loop system is described by a feedback interconnection of a rational transfer function and an unknown, possibly infinite dimensional, dynamical system (see, e.g., [7]-[15]). For example, in the work presented in [14], the present authors have proposed a robust output-feedback MPC design for systems with constraints and unstructured model uncertainty; this procedure minimizes a quadratic upper bound on a nominal cost function, and contains the designs in [16], [17] as special cases (obtained by choosing an associated "pre-stabilizing" feedback gain to be optimal with respect to the nominal cost).

Manuscript received November 04, 2008; revised July 27, 2009, November 09, 2009, and November 13, 2009. First published February 05, 2010; current version published July 08,2010 . This work was supported by Statoil ASA. The numerical examples were implemented using MATLAB with YALMIP [33], SeDuMi [34], MPT [35] and the Matlab Invariant Set Toolbox [36]. Recommended by Associate Editor R. D. Braatz.

C. Løvaas is with Statoil ASA, Kollsnes, Norway (e-mail: chlo@statoil.com).

M. M. Seron and G. C. Goodwin are with the ARC Centre of Excellence for Complex Dynamic Systems and Control (CDSC), The University of Newcastle, Callaghan NSW 2308, Australia (e-mail: maria.seron@newcastle.edu.au; graham.goodwin@newcastle.edu.au).

Digital Object Identifier 10.1109/TAC.2010.2042344
Whilst the MPC designs in, for example, [14], [16]-[18] apply to systems with integrating (and unstable) poles, they do not explicitly incorporate integral action. In particular, these designs require the system model to be stabilizable. Hence, they can not be applied to control the augmented system model obtained when one, for example, utilizes the standard output disturbance model in order to achieve integral action (c.f. [19], [20]). Indeed, in the latter case, it is necessary to steer the original system model towards a time-varying, (generally) non-zero steady-state estimate which accounts for the (generally) non-zero disturbance estimate. Moreover, the objective is usually to converge to a disturbance estimate which depends on both the actual disturbance and the model uncertainty. Hence, to establish robust stability of MPC with integral action, it is necessary to establish robust convergence to a steady-state which depends on uncertain quantities. Such a stability result has, however, to the authors' knowledge, not previously appeared in the literature-at least not in the present context of imperfect state information and dynamic model uncertainty (see, e.g., [19]-[24]).

In the current paper, we present such a stability result for a class of square, open-loop stable systems, having hard constraints, linear-time invariant model uncertainty and non-vanishing output disturbances. The robust MPC design we propose is based on (i) a single quadratic programme (QP) and (ii) a linear estimator utilizing a standard output disturbance model in order to estimate both the output disturbance and the system state. In order to ensure robust closed-loop stability whenever the QP is feasible at the initial time, we impose "tighter" constraints on the predictions, and we choose the cost function parameters off-line so as to satisfy a linear matrix inequality condition.

The paper outline is as follows: Section II establishes notation and sets up the control problem. Section III parameterizes a novel class of robust output-feedback policies. Section IV proposes a procedure which, via an off-line optimization problem, selects an MPC policy within the parameterized class. Numerical examples are presented in Section IV-C [33]-[36]. Concluding remarks are given in Section V. Appendices A and B contain proofs, and Appendix $C$ explains how our results can be generalized to open-loop unstable dynamics.

\section{PRELIMINARIES}

\section{A. Notation and Terminology}

The set of real numbers is denoted $\mathbb{R}$ and the set of non-negative integers is denoted $\mathbb{N}^{+}$. The Hilbert space of all real, squaresummable, one-sided (vector) sequences $\mathbf{z}=\left\{z_{k}\right\}_{k=0}^{\infty}$ is denoted $\ell_{2}$ and the associated extended space is denoted $\ell_{2 e}$ [25]. 


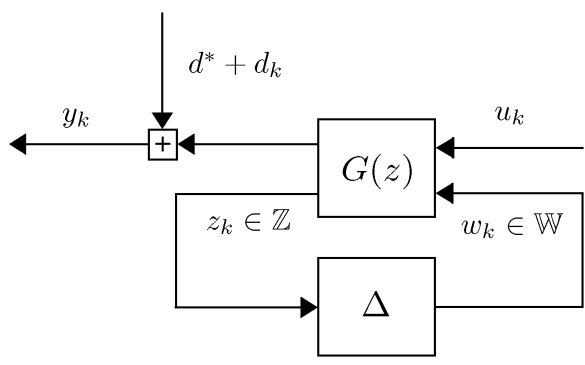

Fig. 1. Uncertain system.

We use $\ell_{2 e}(\mathbb{Z})$ (or $\ell_{2}(\mathbb{Z})$ ) to denote the subset of $\ell_{2 e}$ (or $\ell_{2}$ ) consisting of sequences $\mathbf{z}=\left\{z_{k}\right\}_{k=0}^{\infty}, z_{k} \in \mathbb{R}^{n_{z}}$, which also satisfy $z_{k} \in \mathbb{Z}, \forall k \in \mathbb{N}^{+}$, where $\mathbb{Z} \subseteq \mathbb{R}^{n_{z}}$. Similar to [14], to represent model uncertainty, we use, linear-time invariant (LTI), possibly biased, operators $\Delta: \ell_{2 e}(\mathbb{Z}) \rightarrow \ell_{2 e}(\mathbb{W})$ belonging to the following set:

$$
\Delta(\mathbb{Z}, \mathbb{W}) \triangleq\left\{\Delta \mid \begin{array}{l}
\exists \mathbf{w}^{0} \in \ell_{2} \text { and } \Delta_{\mathrm{T}}(z) \in \Delta_{\mathrm{ESLTI}} \\
\operatorname{such} \text { that }\left\|\Delta_{\mathrm{T}}(z)\right\|_{\infty} \leq 1 \text { and } \\
\Delta \mathbf{z}=\Delta_{\mathrm{T}}(z) \mathbf{z}+\mathbf{w}^{0} \in \ell_{2 e}(\mathbb{W}), \\
\forall \mathbf{z} \in \ell_{2 e}(\mathbb{Z})
\end{array}\right\} .
$$

Here, $\|\cdot\|_{\infty}$ denotes the usual $H_{\infty}$-norm of a transfer function, $\Delta_{\mathrm{T}}(z) \mathbf{z}$ denotes the response of a system with transfer function $\Delta_{\mathrm{T}}(z)$ to the input $\mathbf{z}$, and $\boldsymbol{\Delta}_{\mathrm{ESLTI}}$ denotes the set of all causal transfer functions having an exponentially decaying impulse response, that is, $\Delta_{\mathrm{ESLTI}} \triangleq\left\{\Delta_{\mathrm{T}}(z)=\sum_{k=0}^{\infty} h_{k} z^{-k} \mid \exists a \in\right.$ $\mathbb{R}$ such that $0 \leq a<1$ and $\left.\left\|h_{k}\right\|_{2} \leq a^{k}, \forall k \in \mathbb{N}^{+}\right\}$. Throughout we will use $\Delta_{\mathrm{T}}(z)$ to denote the transfer function associated with a given operator $\Delta \in \Delta(\mathbb{Z}, \mathbb{W})$. Moreover, $[a, \cdots, c]$ denotes $\left[a^{\mathrm{T}} \cdots c^{\mathrm{T}}\right]^{\mathrm{T}}, I_{q}$ (or $I$ ) denotes the identity matrix of dimension $q \times q$ (or of appropriate dimension), $\mathbb{X} \oplus \mathbb{Y}$ denotes the Minkowski sum of the sets $\mathbb{X}$ and $\mathbb{Y}$ (i.e., $\mathbb{X} \oplus \mathbb{Y}=\{x+y \mid x \in \mathbb{X}, y \in \mathbb{Y}\})$ and $\mathbb{X} \sim \mathbb{Y}$ denotes the Pontryagin difference of the sets $\mathbb{X}$ and $\mathbb{Y}$ (i.e., $\mathbb{X} \sim \mathbb{Y}=\{z \mid z+y \in$ $\mathbb{X}, \forall y \in \mathbb{Y}\}$ ). We will also use the following definition:

Definition 2.1: A set $\Omega$ is robustly invariant for the system $x_{k+1}=f\left(x_{k}, w_{k}\right)$ with input $w_{k} \in \mathbb{W}$ (and output $q_{k}=$ $h\left(x_{k}, w_{k}\right) \in \mathbb{Q}$ ) if: $f\left(x_{k}, w_{k}\right) \in \Omega, \forall\left[x_{k}, w_{k}\right] \in \Omega \times \mathbb{W}$ (and, in addition, $\left.h\left(x_{k}, w_{k}\right) \in \mathbb{Q}, \forall\left[x_{k}, w_{k}\right] \in \Omega \times \mathbb{W}\right)$.

\section{B. System Description}

We consider a system of the form shown in Fig. 1, where $d^{*}$ is a constant output disturbance, $d_{k}$ is a vanishing output disturbance, $\Delta \in \boldsymbol{\Delta}(\mathbb{Z}, \mathbb{W})$ is an unknown operator, and where $G(z)$ is a rational transfer function with state-space representation of the form

$$
\begin{aligned}
G(z) & =\left[\begin{array}{ll}
G_{11}(z) & G_{12}(z) \\
G_{21}(z) & G_{22}(z)
\end{array}\right] \\
& =\left[\begin{array}{c}
C \\
C_{z}
\end{array}\right](z I-A)^{-1}\left[\begin{array}{ll}
B & B_{w}
\end{array}\right]+\left[\begin{array}{cc}
0 & 0 \\
D & 0
\end{array}\right] .
\end{aligned}
$$

We use $x_{k} \in \mathbb{R}^{n_{x}}$ to denote the state of the system $G(z)$. The dynamics may then be expressed as follows:

$$
\begin{aligned}
x_{k+1} & =A x_{k}+B u_{k}+B_{w} w_{k}, \quad x_{0}=x \\
y_{k} & =C x_{k}+d^{*}+d_{k} \\
z_{k} & =C_{z} x_{k}+D u_{k}
\end{aligned}
$$

where $u_{k} \in \mathbb{R}^{n}$ is the control input, $y_{k} \in \mathbb{R}^{n}$ is the measured system output and $w_{k} \in \mathbb{W}$ is the response of $\Delta$ to $z_{k} \in \mathbb{Z}$. The system is subject to constraints which we express in state space coordinates as follows:

$$
\begin{aligned}
& q_{k} \in \mathbb{Q}, \quad \forall k \in \mathbb{N}^{+}, \\
& q_{k}=C_{q}\left[x_{k}, u_{k-1}\right]+D_{q} u_{k}, \quad u_{-1}=\overleftarrow{u}
\end{aligned}
$$

Here, $C_{q}, D_{q}$ and $\overleftarrow{u}$ are matrices of appropriate dimension and $\mathbb{Q} \subseteq \mathbb{R}^{n_{q}}$ is a given polyhedral set (with the origin contained in the interior). Note that we consider an "augmented state" $\left[x_{k}, u_{k-1}\right]$ in (4), so as to allow for the possible inclusion of rate constraints on the control input. Furthermore, the constraints (4) are consistent with the domain of $\Delta$, and the output disturbances are contained in some given sets, in the sense that the following standing assumptions hold:

Assumption 2.1: We have the implication that $C_{q}\left[x, u_{-1}\right]+$ $D_{q} u \in \mathbb{Q} \Rightarrow C_{z} x+D u \in \mathbb{Z}$.

Assumption 2.2: For given convex sets $\mathbb{D}^{*} \subseteq \mathbb{R}^{n}, \mathbb{D} \subseteq \mathbb{R}^{n}$, we have $d^{*} \in \mathbb{D}^{*}$ and $\left\{d_{k}\right\} \in \ell_{2}(\mathbb{D})$.

\section{Problem Formulation}

The (main) goal of the MPC design considered here is to steer the measured output $y_{k}$ to zero whilst respecting the given constraints. To this end, we shall assume that (i) the system is square (c.f. $u_{k} \in \mathbb{R}^{n}, y_{k} \in \mathbb{R}^{n}$ ) and that (ii) the system matrix $A$ is stable. ${ }^{1}$ We also require $\Delta$ to be such that the open-loop system transfer function

$G_{\Delta}(z) \triangleq G_{11}(z)+G_{12}(z) \Delta_{\mathrm{T}}(z)\left(I-G_{22}(z) \Delta_{\mathrm{T}}(z)\right)^{-1} G_{21}(z)$

is well-defined and invertible at one. That is, we define the set of admissible perturbations $\Delta: \ell_{2 e}(\mathbb{Z}) \rightarrow \ell_{2 e}(\mathbb{W})$ as follows:

$$
\Delta^{+}(\mathbb{Z}, \mathbb{W}) \triangleq\left\{\Delta \in \Delta(\mathbb{Z}, \mathbb{W}) \mid G_{\Delta}(1) \text { is full rank }\right\}
$$

where the set $\boldsymbol{\Delta}(\mathbb{Z}, \mathbb{W})$ is as defined in (1). The invertibility of $G_{\Delta}(1)$ allows us to define "setpoints" for $u_{k}, z_{k}, w_{k}$ and $x_{k}$, which correspond to $y_{k}=0$, and depend on the uncertain/ unknown operator $\Delta$, as follows:

$$
\begin{aligned}
\mathbf{u}^{*}(\Delta) & \triangleq-\left[G_{\Delta}(1)\right]^{-1} d^{*} \\
\mathbf{z}^{*}(\Delta) & \triangleq\left[I-G_{22}(1) \Delta_{\mathrm{T}}(1)\right]^{-1} G_{21}(1) \mathbf{u}^{*}(\Delta) \\
\mathbf{w}^{*}(\Delta) & \triangleq \Delta_{\mathrm{T}}(1) \mathbf{z}^{*}(\Delta) \\
\mathbf{x}^{*}(\Delta) & \triangleq[I-A]^{-1}\left[B \mathbf{u}^{*}(\Delta)+B_{w} \mathbf{w}^{*}(\Delta)\right] .
\end{aligned}
$$

The proposed MPC design is aimed at robustly stabilizing the system in the following sense:

Definition 2.2: A causal control policy (based on the measured output $y_{k}$ ) is said to robustly stabilize the system (3) if the following holds for the closed-loop signals:

$$
\begin{gathered}
\left\{x_{k}-\mathbf{x}^{*}(\Delta)\right\}_{k=0}^{\infty} \in \ell_{2}, \quad\left\{u_{k}-\mathbf{u}^{*}(\Delta)\right\}_{k=0}^{\infty} \in \ell_{2}, \\
\{q\}_{k=0}^{\infty} \in \ell_{2 e}(\mathbb{Q})
\end{gathered}
$$

for all $\Delta \in \Delta^{+}(\mathbb{Z}, \mathbb{W})$.

\footnotetext{
${ }^{1}$ As explained in Appendix C, our results extend to the general case when $A$ has no eigenvalues equal to one.
} 
Remark 2.1: In view of (5), we may interpret $G_{11}(z)$ as the nominal model of the system. The assumption $\Delta \in \Delta^{+}(\mathbb{Z}, \mathbb{W})$ together with the transfer functions $G_{12}(z), G_{22}(z), G_{21}(z)$ constitute a description of the model uncertainty associated with $G_{11}(z)$.

Remark 2.2: Note that our problem formulation covers the problem of tracking a given nonzero setpoint, say $y_{k}=y^{*}$, provided we make a simple re-definition of the system output and the constant output disturbance, that is, we consider $y_{k} \leftarrow$ $y_{k}-y^{*}$ and $d^{*} \leftarrow d^{*}-y^{*}$. Also, to satisfy Assumption 2.2, we use $\mathbb{D}^{*} \leftarrow \mathbb{D}^{*} \oplus\left\{-y^{*}\right\}$.

\section{A Class of Robust MPC Policies}

In this section we parameterize a class of robustly stabilizing MPC policies; each policy within the class satisfies a robust stability test which we present in Section III-E.

\section{A. The State- and Steady-State Target Estimator}

Consider the following linear state estimator based on the standard output disturbance model (see [19], [20], [26]):

$$
\left[\hat{x}_{k+1}, \hat{d}_{k+1}\right]=A_{L}\left[\hat{x}_{k}, \hat{d}_{k}\right]+\left[\begin{array}{c}
B \\
0
\end{array}\right] u_{k}+\left[\begin{array}{c}
L_{1} \\
L_{2}
\end{array}\right] y_{k}
$$

where $\left[\hat{x}_{0}, \hat{d}_{0}\right]=[\hat{x}, \hat{d}]$

$$
A_{L} \triangleq\left[\begin{array}{cc}
A & 0 \\
0 & I
\end{array}\right]-\left[\begin{array}{l}
L_{1} \\
L_{2}
\end{array}\right]\left[\begin{array}{ll}
C & I
\end{array}\right]
$$

and where $\left[L_{1}, L_{2}\right]$ is an appropriately selected observer gain such that $A_{L}$ is stable (i.e., all its eigenvalues are strictly inside the unit disk). The dynamics of the associated estimation error, that is

$$
e_{k} \triangleq\left[x_{k}-\hat{x}_{k}, d^{*}-\hat{d}_{k}\right]
$$

are

$$
e_{k+1}=A_{L} e_{k}+\left[\begin{array}{cc}
B_{w} & -L_{1} \\
0 & -L_{2}
\end{array}\right]\left[\begin{array}{l}
w_{k} \\
d_{k}
\end{array}\right]
$$

To enable robust constraint satisfaction we will require the following assumption.

Co We have available a (convex) set $\mathbb{E}$ which is robustly invariant for the system (12) with input $\left[w_{k}, d_{k}\right] \in \mathbb{W} \times \mathbb{D}$. In the sequel we shall assume that the estimation error is contained within the invariant set $\mathbb{E}$. However, the error will not necessarily converge to zero. Specifically, its (desired) steady state value is [c.f. (7c)]

$$
\begin{aligned}
\mathbf{e}^{*}(\Delta) & \triangleq\left(I-A_{L}\right)^{-1}\left[\begin{array}{c}
B_{w} \\
0
\end{array}\right] \mathbf{w}^{*}(\Delta) \\
& =\left[\begin{array}{c}
(I-A)^{-1} B_{w} \mathbf{w}^{*}(\Delta) \\
-C(I-A)^{-1} B_{w} \mathbf{w}^{*}(\Delta)
\end{array}\right] .
\end{aligned}
$$

Accordingly, it will be convenient to introduce the auxiliary variable

$$
e_{k}^{\mathrm{d}} \triangleq e_{k}-\mathrm{e}^{*}(\Delta)
$$

with dynamics

$$
e_{k+1}^{\mathrm{d}}=A_{L} e_{k}^{\mathrm{d}}+\left[\begin{array}{cc}
B_{w} & -L_{1} \\
0 & -L_{2}
\end{array}\right]\left[\begin{array}{l}
w_{k}^{\mathrm{d}} \\
d_{k}
\end{array}\right]
$$

where $w_{k}^{\mathrm{d}} \triangleq w_{k}-\mathbf{w}^{*}(\Delta)$.

At each time step, the MPC policy uses the estimate $\hat{d}_{k}$ obtained from (9) to compute the following estimates of, respectively, the steady-state control input and the steady-state state estimate:

$$
\begin{aligned}
& \hat{u}_{k}^{*}=-\left[C(I-A)^{-1} B\right]^{1} \hat{d}_{k} \\
& \hat{x}_{k}^{*}=(I-A)^{-1} B \hat{u}_{k}^{*} .
\end{aligned}
$$

An adjustment $v_{k}^{*}$ of $\hat{u}_{k}^{*}$ is then calculated by optimizing a criterion (to be introduced below). In closed-loop we have

$$
u_{k}=\hat{u}_{k}^{*}+v_{k}^{*}=-\left[C(I-A)^{-1} B\right]^{-1} \hat{d}_{k}+v_{k}^{*}
$$

where $v_{k}^{*}$ is determined by the MPC policy. For later reference we introduce the following variables, which we shall make frequent use of in our closed-loop analysis:

$$
\begin{aligned}
z_{k}^{\mathrm{d}} & \triangleq z_{k}-\mathbf{z}^{*}(\Delta) \\
c_{k} & \triangleq\left[\begin{array}{c}
\hat{x}_{k} \\
u_{k-1}
\end{array}\right] \\
c_{k}^{\mathrm{d}} & \triangleq c_{k}-\left[\begin{array}{c}
\hat{x}_{k}^{*} \\
\hat{u}_{k}^{*}
\end{array}\right]=c_{k}-K_{1} \hat{d}_{k} \\
K_{1} & \triangleq\left[\begin{array}{c}
\left(I-A^{-1} B\right. \\
I
\end{array}\right]\left[C(I-A)^{-1} B\right]^{-1} .
\end{aligned}
$$

The dynamics of the various variables introduced above are then, as shown in Appendix A

$$
\begin{aligned}
& {\left[\begin{array}{c}
e_{k+1}^{\mathrm{d}} \\
c_{k+1}^{\mathrm{d}}
\end{array}\right]=\underbrace{\left[\begin{array}{cc}
A_{L} & 0 \\
\Lambda_{1} & A_{0}
\end{array}\right]}_{A_{a}}\left[\begin{array}{c}
e_{k}^{\mathrm{d}} \\
c_{k}^{\mathrm{d}}
\end{array}\right]+\underbrace{\left[\begin{array}{c}
0 \\
B_{I}
\end{array}\right]}_{B_{\mathrm{a}}} v_{k}^{*}+\underbrace{\left.\left[\begin{array}{c}
B_{w} \\
0 \\
0
\end{array}\right]\right]}_{B_{\mathrm{a}, w}} w_{k}^{\mathrm{d}}} \\
& +\underbrace{\left.\left[\begin{array}{c}
-L_{1} \\
L_{2} \\
\Lambda_{2}
\end{array}\right]\right]}_{B_{\mathrm{a}}, d} d_{k} \\
& z_{k}^{\mathrm{d}}=\underbrace{\left[\begin{array}{lll}
\Lambda_{3} & {\left[\begin{array}{ll}
C_{z} & 0
\end{array}\right]}
\end{array}\right]}_{C_{\mathrm{a}, z}}\left[\begin{array}{c}
e_{k}^{\mathrm{d}} \\
c_{k}^{\mathrm{d}}
\end{array}\right]+D v_{k}^{*}
\end{aligned}
$$

where

$$
\begin{aligned}
& A_{0} \triangleq\left[\begin{array}{cc}
A & 0 \\
0 & 0
\end{array}\right], \quad B_{I} \triangleq\left[\begin{array}{c}
B \\
I
\end{array}\right] \\
& \Lambda_{1} \triangleq\left(\left[\begin{array}{c}
L_{1} \\
0
\end{array}\right]-K_{1} L_{2}\right)\left[\begin{array}{ll}
C & I
\end{array}\right] \\
& \Lambda_{2} \triangleq\left(\left[\begin{array}{c}
L_{1} \\
0
\end{array}\right]-K_{1} L_{2}\right) \\
& \Lambda_{3} \triangleq C_{z} G_{1}+D G_{2} \\
& G_{1} \triangleq\left[\begin{array}{ll}
I & (I-A)^{-1} B\left[C(I-A)^{-1} B\right]^{-1}
\end{array}\right], \\
& G_{2} \triangleq\left[\begin{array}{ll}
0 & {\left[C(I-A)^{-1} B\right]^{-1}}
\end{array}\right] .
\end{aligned}
$$




\section{B. The Candidate Policy}

As in [14], the MPC policies we consider "inherit" their robustness properties from an associated candidate policy, namely, the policy given by $v_{k}^{*}=0$. Hence, we require the closed-loop system obtained by use of $u_{k}=\hat{u}_{k}^{*}$ (c.f. (18)) to be robustly stable in the sense that it satisfies the following small-gain condition (c.f. the Small-Gain Theorem, [25]):

C1 We have $\left\|C_{\mathrm{a}, z}\left(z I-A_{\mathrm{a}}\right)^{-1} B_{\mathrm{a}, w}\right\|_{\infty}<1$, where the various matrices are as in (23). ${ }^{2}$

To enable selection of the constraints to be imposed over the prediction horizon, we also require that there exist appropriate (invariant) constraint sets. This condition is called $\mathbf{C 2}$ and is expressed next using the matrices of the following system:

$$
\begin{aligned}
{\left[\begin{array}{c}
c_{k+1} \\
\hat{u}_{k+1}^{*}
\end{array}\right]=} & \underbrace{\left[\begin{array}{cc}
A_{0} & B_{I} \\
0 & I
\end{array}\right]}_{\hat{A}}\left[\begin{array}{c}
c_{k} \\
\hat{u}_{k}^{*}
\end{array}\right]+\left[\begin{array}{c}
B_{I} \\
0
\end{array}\right] v_{k}^{*} \\
& +\underbrace{\left[\begin{array}{c}
L_{1} \\
0
\end{array}\right]}_{\hat{B}} \begin{array}{c}
{\left[C(I-A)^{-1} B\right]^{-1} L_{2}}
\end{array}]\left[\begin{array}{lll}
C & I & I
\end{array}\right] \\
& \times\left[\begin{array}{l}
e_{k} \\
d_{k}
\end{array}\right] \\
\hat{q}_{k}= & \underbrace{\left[\begin{array}{ll}
C_{q} & D_{q}
\end{array}\right]}_{\hat{C}}\left[\begin{array}{l}
c_{k} \\
\hat{u}_{k}^{*}
\end{array}\right]+D_{q} v_{k}^{*} .
\end{aligned}
$$

C2 We have available a non-empty (polyhedral) set $\mathcal{R} \subseteq$ $\mathbb{R}^{n_{x}}$ which is robustly invariant for the system

$$
c_{i+1}=A_{0} c_{i}+A_{0}^{\left(N_{\mathrm{s}}-1\right)}\left[\Lambda_{1} \Lambda_{2}\right]\left[e_{i}, d_{i}\right]
$$

with input $\left[e_{i}, d_{i}\right] \in \mathbb{E}_{\mathbb{D}}$ and output $C_{q} c_{i} \in \hat{\mathbb{Q}}_{N_{s}-1}^{\mathrm{d}}$, where

$$
\begin{aligned}
\hat{\mathbb{Q}}_{N_{s}-1}^{\mathrm{d}} & \triangleq \hat{\mathbb{Q}}_{N_{s}-1} \sim K_{2} \hat{\mathbb{D}} \\
\hat{\mathbb{Q}}_{i+1} & \triangleq \hat{\mathbb{Q}}_{i} \sim \hat{C} \hat{A}^{i} \hat{B} \mathbb{E}_{\mathbb{D}}, \\
\forall i & \in\left\{0, \cdots, N_{\mathrm{s}}-2\right\} \\
\mathbb{E}_{\mathbb{D}} & \triangleq \mathbb{E} \times \mathbb{D}, \quad \hat{\mathbb{Q}}_{0} \triangleq \mathbb{Q} \sim C_{q}\left[\begin{array}{ll}
I & 0 \\
0 & 0
\end{array}\right] \mathbb{E} \\
K_{2} & \triangleq\left(C_{q}+\left[\begin{array}{ll}
0 & D_{q}
\end{array}\right]\right) K_{1} \\
\hat{\mathbb{D}} & \triangleq \mathbb{D}^{*} \oplus[0-I] \mathbb{E} .
\end{aligned}
$$

Here, $N_{\mathrm{s}}$ denotes a selectable integer satisfying $N_{\mathrm{s}} \geq 1, \mathbb{Q}$ is as in (4), $\mathbb{D}^{*}$ and $\mathbb{D}$ are as in Assumption 2.2 and $\mathbb{E}$ is as in CO. In (31), the matrices are as in (23).

Remark 3.1: Note that (33), (34) constitute a well-known constraint tightening recursion for the system (30) (c.f. [17], [27]), and that, subject to C0, we have $\hat{d}_{k} \in \hat{\mathbb{D}}, \forall k \in \mathbb{N}^{+}$. The integer $N_{\mathrm{s}}$ and the above stated requirements on the set $\mathcal{R}$ are amongst the novel features introduced in this paper. As will become clearer below, their purpose is to allow us to adapt the approach in [27] and impose suitably selected constraints on the $\left(N_{\mathrm{s}}+i\right)$-step predicted value of $\hat{q}_{k}^{\mathrm{d}} \triangleq \hat{q}_{k}-K_{2} \hat{d}_{k}$ (see (30b), (35) and (42), (43) below). Note that, once the integer $N_{\mathrm{s}} \geq 1$

\footnotetext{
${ }^{2}$ Note that $A_{\mathrm{a}}$ is stable by our choice of $L$.
}

has been selected such that the resulting set $\hat{\mathbb{Q}}_{N_{s}-1}^{\mathrm{d}}$ contains the origin (in its interior), then it is a linear programming problem to compute the set $\mathcal{R}$ as the maximal set which satisfies the above conditions (see, e.g., [28]). Further comments on the integer $N_{\mathrm{s}}$ are given in Remark 3.3.

Remark 3.2 (Choosing L Subject to C0-C2): It is important to note that any stabilizing choice of $L$ will lead to satisfaction of conditions C0-C2 provided the sets $\mathbb{W}$ and $\mathbb{D}$ (and hence the set $\mathbb{E})$ and the transfer matrices $G_{21}(z)$ and $G_{22}(z)$ are sufficiently small (i.e., whenever the uncertain quantities are sufficiently small). Also note that choosing $L$ subject to $\mathbf{C 1}$ is a standard $H_{\infty}$ static output feedback gain problem. For example, it is possible to compute $L$ and "small" ellipsoids $\mathbb{E}$ satisfying both $\mathbf{C O}$ and $\mathbf{C 1}$ using convex optimization, by following the approach to multi-objective linear control proposed in [29], and similarly in Chapter 3.6 of [13].

\section{The Constraints}

At each time step, the prediction based control policy is parameterized as an $N$-step open-loop prediction of the candidate policy $u_{k}=\hat{u}_{k}^{*}$, that is

$$
u_{k+i}=\hat{u}_{k}^{*}+v_{i \mid k}, \forall i \geq 0, \quad v_{i \mid k}=0, \forall i \geq N
$$

where $u_{k+i}$ is the predicted control input, and where $v_{0 \mid k}, \cdots, v_{N-1 \mid k}$ are the "degrees of freedom" in the optimization problem. In the sequel we use $\mu \triangleq\left[v_{0 \mid k}, \cdots, v_{N-1 \mid k}\right]$ to denote the degrees of freedom, and we impose the following (tightened) constraints on the predicted nominal trajectory, in order to ensure both constraint satisfaction and recursive feasibility:

$$
\begin{aligned}
c_{i+1 \mid k}^{\mathrm{d}} & =A_{0} c_{i \mid k}^{\mathrm{d}}+B_{I} v_{i \mid k}, \quad c_{0 \mid k}^{\mathrm{d}}=c_{k}^{\mathrm{d}} \\
\hat{q}_{i \mid k}^{\mathrm{d}} & =C_{q} c_{i \mid k}^{\mathrm{d}}+D_{q} v_{i \mid k} \\
\hat{q}_{i \mid k} & =\hat{q}_{i \mid k}^{\mathrm{d}}+K_{2} \hat{d}_{k} \\
\hat{q}_{i \mid k} & \in \hat{\mathbb{Q}}_{i}, \quad \forall i \in\left\{0, \cdots, N_{\mathrm{s}}-1\right\} \\
\hat{q}_{i \mid k}^{\mathrm{d}} & \in \hat{\mathbb{Q}}_{i}^{\mathrm{d}}, \quad \forall i \in\left\{N_{\mathrm{s}}, \cdots, N-1\right\} \\
c_{N \mid k}^{\mathrm{d}} & \in \mathbb{X}_{f}
\end{aligned}
$$

where $c_{k}^{\mathrm{d}}$ is as in (21), $N$ is the control prediction horizon satisfying $N \geq N_{\mathrm{s}}$

$$
\begin{aligned}
& \hat{\mathbb{Q}}_{i+1}^{\mathrm{d}} \triangleq \hat{\mathbb{Q}}_{i}^{\mathrm{d}} \sim C_{q} A_{0}^{i}\left[\Lambda_{1} \Lambda_{2}\right] \mathbb{E}_{\mathbb{D}} \\
& \forall i \in\left\{N_{\mathrm{s}}-1, \cdots, N-2\right\} \\
& \mathbb{X}_{f} \triangleq \mathcal{R} \sim\left(A_{0}^{N_{s}-1}\left[\Lambda_{1} \Lambda_{2}\right] \mathbb{E}_{\mathbb{D}} \oplus \cdots\right. \\
&\left.\oplus A_{0}^{N-1}\left[\Lambda_{1} \Lambda_{2}\right] \mathbb{E}_{\mathbb{D}}\right)
\end{aligned}
$$

and where the integer $N_{\mathrm{s}}$ and the sets, $\hat{\mathbb{Q}}_{i}, \hat{\mathbb{Q}}_{N_{\mathrm{s}}-1}^{\mathrm{d}}, \mathbb{E}_{\mathbb{D}}$ and $\mathcal{R}$, are as in $\mathbf{C 2}$.

Remark 3.3 (Choosing $N_{\mathrm{s}} \geq 1$ ): It can be shown that if $\mathbf{C 2}$ holds using $N_{\mathrm{s}} \geq 2$ and $\mathcal{R}$, then $\mathbf{C} 2$ also holds using $N_{\mathrm{s}} \leftarrow N_{\mathrm{S}}-$ 1 and $\mathcal{R} \leftarrow\left\{c \mid \bar{A}_{0} c \in \mathcal{R}\right\}$. Thus, to identify all possible choices of $N_{\mathrm{s}}$ (given $N$ and the set $\mathbb{E}$ ), we may increment $N_{\mathrm{s}} \leq N$ until there does not exist a corresponding invariant set $\mathcal{R}$ (e.g., because the set $\hat{\mathbb{Q}}_{N_{s}-1}^{\mathrm{d}}$ is empty). 
Note that the various constraint sets and Pontryagin differences are polyhedral and may be computed efficiently. Hence the above constraints (38)-(43) may be expressed as follows:

$$
S_{0}\left[c_{k}^{\mathrm{d}}, \mu\right]+S_{1} \hat{d}_{k} \leq s
$$

using $\mu=\left[v_{0 \mid k}, \cdots, v_{N-1 \mid k}\right]$ and some matrices, $S_{0}, S_{1}$ and $s$. In what follows, we will frequently write these constraints as $\left[c_{k}^{\mathrm{d}}, \mu\right] \in \mathbb{S}\left(\hat{d}_{k}\right)$ using the set-valued map:

$$
\mathbb{S}\left(\hat{d}_{k}\right) \triangleq\left\{y \mid S_{0} y \leq s-S_{1} \hat{d}_{k}\right\} .
$$

As shown in the following theorem, the set $\mathbb{S}\left(\hat{d}_{k}\right)$ has an interesting invariance property which, amongst other things, guarantees robust recursive feasibility.

Theorem 3.1 (Robust Recursive Feasibility): Let $\mathbf{C 2}$ hold and consider any $\hat{d}_{t} \in \hat{\mathbb{D}}$ and any $\left[e_{t}, d_{t}\right] \in \mathbb{E}_{\mathbb{D}}$ such that

$$
\hat{d}_{t+1}=\hat{d}_{t}+L_{2}\left(\left[\begin{array}{ll}
C & I
\end{array}\right] e_{t}+d_{t}\right) \in \hat{\mathbb{D}}
$$

where the set $\hat{\mathbb{D}}$ is as defined in (36), then, the following holds:

$$
\begin{aligned}
{\left[\begin{array}{c}
c_{t+1}^{\mathrm{d}} \\
\mu_{t+1}
\end{array}\right] } & =\left[\begin{array}{cc}
A_{0} & B_{I} D_{1} \\
0 & \Gamma
\end{array}\right]\left[\begin{array}{l}
c_{t}^{\mathrm{d}} \\
\mu_{t}
\end{array}\right]+\left[\begin{array}{cc}
\Lambda_{1} & \Lambda_{2} \\
0 & 0
\end{array}\right]\left[\begin{array}{c}
e_{t} \\
d_{t}
\end{array}\right] \\
& \in \mathbb{S}\left(\hat{d}_{t+1}\right), \quad \forall\left[\begin{array}{c}
c_{t}^{\mathrm{d}} \\
\mu_{t}
\end{array}\right] \in \mathbb{S}\left(\hat{d}_{t}\right)
\end{aligned}
$$

where $D_{1} \triangleq\left[\begin{array}{llll}I_{n} & 0 & \cdots & 0\end{array}\right] \in \mathbb{R}^{n \times N n}$ and

$$
\Gamma \triangleq\left[\begin{array}{cc}
0 & I_{(N-1) n \times(N-1) n} \\
0 & 0
\end{array}\right] \in \mathbb{R}^{N n \times N n} .
$$

Proof: We need to show that, if $\mu=\mu_{t}$ is feasible at time $k=t$, and $u_{k}=\hat{u}_{k}^{*}+D_{1} \mu_{t},\left[e_{k}, d_{k}\right] \in \mathbb{E}_{\mathbb{D}}$, then choosing $\mu=\Gamma \mu_{t}=\left[v_{1 \mid t}, \cdots, v_{N-1 \mid t}, 0\right]$ is feasible at time $k=t+1$. To this end, we express the trajectories obtained in (38)-(43) at time $k=t+1$ as a perturbation of the trajectories obtained at time $k=t$, as follows:

$$
\begin{aligned}
\hat{q}_{i \mid t+1}= & \hat{q}_{i+1 \mid t}+\hat{C} \hat{A}^{i} \hat{B}\left[e_{t}, d_{t}\right] \\
& \forall i \in\left\{0, \cdots, N_{s}-2\right\} \\
\hat{q}_{N_{s}-1 \mid t+1}= & \hat{q}_{N_{s} \mid t}^{\mathrm{d}}+C_{q} A_{0}^{N_{s}-1}\left[\Lambda_{1} \Lambda_{2}\right]\left[e_{t}, d_{t}\right]+K_{2} \hat{d}_{t+1} \\
\hat{q}_{i \mid t+1}^{\mathrm{d}}= & \hat{q}_{i+1 \mid t}^{\mathrm{d}}+C_{q} A_{0}^{i}\left[\Lambda_{1} \Lambda_{2}\right]\left[e_{t}, d_{t}\right] \\
\forall i & \in\left\{N_{\mathrm{s}}, \cdots, N-2\right\} \\
\hat{q}_{N-1 \mid t+1}^{\mathrm{d}}= & C_{q}\left(c_{N \mid t}^{\mathrm{d}}+A_{0}^{N-1}\left[\Lambda_{1} \Lambda_{2}\right]\left[e_{t}, d_{t}\right]\right) \\
c_{N \mid t+1}^{\mathrm{d}}= & A_{0}\left(c_{N \mid t}^{\mathrm{d}}+A_{0}^{N-1}\left[\Lambda_{1} \Lambda_{2}\right]\left[e_{t}, d_{t}\right]\right) .
\end{aligned}
$$

Feasibility at time $k=t$ implies $\hat{q}_{i+1 \mid t} \in \hat{\mathbb{Q}}_{i}, \forall i \in\left\{1, \cdots, N_{\mathrm{s}}-\right.$ $2\}$, and $\hat{q}_{N_{s} \mid t}^{\mathrm{d}} \in \hat{\mathbb{Q}}_{N_{s}}^{\mathrm{d}}$, and thus it follows from (32)-(34), (44), (50), (51), $\left[e_{t}, d_{t}\right] \in \mathbb{E}_{\mathbb{D}}$ and $\hat{d}_{t+1} \in \hat{\mathbb{D}}$ that $\hat{q}_{i \mid t+1} \in \hat{\mathbb{Q}}_{i}, \forall i \in$ $\left\{0, N_{\mathrm{s}}-1\right\}$ (i.e., the constraints (41) hold at time $k=t+1$ using $\mu=\Gamma \mu_{t}$ ). Similarly, feasibility at time $k=t$ implies $\hat{q}_{i+1 \mid t}^{\mathrm{d}} \in \hat{\mathbb{Q}}_{i}^{\mathrm{d}}, \forall i \in\left\{N_{\mathrm{s}}, \cdots, N-2\right\}$, and $C_{q} c_{N \mid t}^{\mathrm{d}} \in \hat{\mathbb{Q}}_{N-1}^{\mathrm{d}} \sim$
$C_{q} A_{0}^{N-1}\left[\Lambda_{1} \Lambda_{2}\right] \mathbb{E}_{\mathbb{D}}$, and thus it follows from (44) and (52), (53) that $\hat{q}_{i \mid t+1}^{\mathrm{d}} \in \hat{\mathbb{Q}}_{i}^{\mathrm{d}}, \forall i \in\left\{N_{\mathrm{s}}, \cdots, N-1\right\}$. To see that the constraint $c_{N \mid t+1}^{\mathrm{d}} \in \mathbb{X}_{f}$ is satisfied, we use (54), $c_{N \mid t}^{\mathrm{d}} \in \mathbb{X}_{f}$ and the fact that the terminal constraint set, $\mathbb{X}_{f}$, is robustly invariant for the system $c_{t+1}=A_{0} c_{t}+A_{0}^{N}\left[\Lambda_{1} \Lambda_{2}\right]\left[e_{t}, d_{t}\right]$ with input $\left[e_{t}, d_{t}\right] \in \mathbb{E}_{\mathbb{D}}$. This invariance property follows from $\mathbf{C 2}$ as can be seen by using properties of the Pontryagin difference [28] as follows:

$$
\begin{aligned}
A_{0} \mathbb{X}_{f}= & A_{0}\left[\mathcal { R } \sim \left(A_{0}^{N_{s}-1}\left[\Lambda_{1} \Lambda_{2}\right] \mathbb{E}_{\mathbb{D}} \oplus \cdots\right.\right. \\
& \left.\left.\oplus A_{0}^{N-1}\left[\Lambda_{1} \Lambda_{2}\right] \mathbb{E}_{\mathbb{D}}\right)\right] \\
= & A_{0} \mathcal{R} \sim\left(A_{0}^{N_{s}}\left[\Lambda_{1} \Lambda_{2}\right] \mathbb{E}_{\mathbb{D}} \oplus \cdots \oplus A_{0}^{N}\left[\Lambda_{1} \Lambda_{2}\right] \mathbb{E}_{\mathbb{D}}\right) \\
& \subseteq\left[\mathcal{R} \sim A_{0}^{N_{s}-1}\left[\Lambda_{1} \Lambda_{2}\right] \mathbb{E}_{\mathbb{D}}\right] \\
& \sim\left(A_{0}^{N_{s}}\left[\Lambda_{1} \Lambda_{2}\right] \mathbb{E}_{\mathbb{D}} \oplus \cdots \oplus A_{0}^{N}\left[\Lambda_{1} \Lambda_{2}\right] \mathbb{E}_{\mathbb{D}}\right) \\
= & \mathbb{X}_{f} \sim A_{0}^{N}\left[\Lambda_{1} \Lambda_{2}\right] \mathbb{E}_{\mathbb{D}} .
\end{aligned}
$$

\section{The Cost Function and the LMI Condition}

Similar to [14], we consider a cost function of the following quadratic form:

$$
J\left(c_{k}^{\mathrm{d}}, \mu\right) \triangleq\left\|\left[c_{k}^{\mathrm{d}}, \mu\right]\right\|_{P}^{2}
$$

where $P$ is some positive definite matrix satisfying the following LMI condition.

Condition 3.1: The cost function matrix $P$ is such that, for some scalar $m \geq 0$, and some symmetric matrix $\Omega_{0} \in \mathbb{R}^{2\left(n_{x}+n\right) \times 2\left(n_{x}+n\right)}$, the following holds:

$$
\boldsymbol{\Phi}\left(\Omega_{0}, P, m\right)<0, \quad \boldsymbol{\Omega}\left(\Omega_{0}, P\right) \geq 0
$$

where

$$
\begin{aligned}
\boldsymbol{\Phi}\left(\Omega_{0}, P, m\right)= & {\left[\begin{array}{cc}
A_{\mathrm{p}} & B_{\mathrm{p}} \\
C_{\mathrm{p}} & 0
\end{array}\right]^{\mathrm{T}}\left[\begin{array}{cc}
\boldsymbol{\Omega}\left(\Omega_{0}, P\right) & 0 \\
0 & m I_{n_{z}}
\end{array}\right]\left[\begin{array}{cc}
A_{\mathrm{p}} & B_{\mathrm{p}} \\
C_{\mathrm{p}} & 0
\end{array}\right] } \\
& -\left[\begin{array}{cc}
\boldsymbol{\Omega}\left(\Omega_{0}, P\right) & 0 \\
0 & m I_{n_{w}}
\end{array}\right] \\
\boldsymbol{\Omega}\left(\Omega_{0}, P\right)= & {\left[\begin{array}{cc}
\Omega_{0} & 0 \\
0 & 0
\end{array}\right]+D_{P}^{\mathrm{T}} P D_{P} } \\
D_{P}= & {\left[\begin{array}{ccc}
0 & I_{\left(n_{x}+n\right)} & 0 \\
0 & 0 & I_{N n}
\end{array}\right] }
\end{aligned}
$$

and where [see (23)]

$$
A_{\mathrm{p}} \triangleq\left[\begin{array}{cc}
A_{\mathrm{a}} & B_{\mathrm{a}} D_{1} \\
0 & \Gamma
\end{array}\right], B_{\mathrm{p}} \triangleq\left[\begin{array}{c}
B_{\mathrm{a}}, w \\
0
\end{array}\right], C_{\mathrm{p}} \triangleq\left[\begin{array}{ll}
C_{\mathrm{a}}, z & D D_{1}
\end{array}\right]
$$

with $D_{1}$ and $\Gamma$ as defined in Theorem 3.1.

Regarding the existence of a matrix $P$ satisfying the above condition we have the following result, which may be proven as in [14]:

Theorem 3.2: There exists a $P$ satisfying Condition 3.1 if and only if $\mathbf{C} 1$ holds. 
In Section IV, we propose a semi-definite program (SDP) which may be used to compute a matrix $P=P^{*}$ that satisfies Condition 3.1 and is as "close" as possible to the matrix $P=P_{\left\{Q, R, R^{\mathrm{d}}\right\}}$ of the following nominal cost:

$$
\begin{aligned}
J\left(\left[x_{0}, u_{-1}\right], \mu\right) & =\left\|\left[x, u_{-1}, \mu\right]\right\|_{P_{\left\{Q, R, R^{\mathrm{d}}\right\}}}^{2} \\
& =\sum_{i=0}^{\infty}\left\|x_{i}\right\|_{Q}^{2}+\left\|u_{i-1}\right\|_{R}^{2}+\left\|\delta u_{i}\right\|_{R^{\mathrm{d}}}^{2}
\end{aligned}
$$

subject to $x_{i+1}=A x_{i}+B u_{i}, u_{i}=v_{i}, \delta u_{i}=v_{i}-u_{i-1}$, $\forall i \geq 0, v_{i}=0, \forall i \geq N$, where $\mu=\left[v_{0}, \cdots, v_{N-1}\right]$, and where $Q \geq 0, R \geq 0$ and $R^{\mathrm{d}}>0$ are selectable weighting matrices. We also show that such a matrix $P^{*}$ may be chosen to satisfy $P^{*} \approx P_{\left\{Q, R, R^{\mathrm{d}}\right\}}$, provided model uncertainty is "sufficiently small" (Theorem 4.1).

\section{E. The Robust MPC Policy}

The resulting robust MPC policy is as follows:

Algorithm 3.1: At time $k \in \mathbb{N}^{+}$, given $u_{k-1}, y_{k}$ and $\left[\hat{x}_{k}, \hat{d}_{k}\right]$, compute (16), (21)

$$
\begin{aligned}
K\left(\left[c_{k}^{\mathrm{d}}, \hat{d}_{k}\right]\right) & \triangleq \arg \min _{\mu \in \mathbf{R}^{N n}}\left\{\left\|\left[c_{k}^{\mathrm{d}}, \mu\right]\right\|_{P}^{2} \text { s.t. }\left[c_{k}^{\mathrm{d}}, \mu\right] \in \mathbb{S}\left(\hat{d}_{k}\right)\right\}, \\
u_{k} & =\hat{u}_{k}^{*}+D_{1} K\left(\left[c_{k}^{\mathrm{d}}, \hat{d}_{k}\right]\right)
\end{aligned}
$$

and update the estimator using (9). Then apply $u_{k}$ to the system (3).

Note that the above is a standard QP with $N n$ degrees of freedom and that the online computational requirements are thus similar to conventional MPC. The following stability test shows that the MPC policy robustly stabilizes the system provided the QP is feasible at the initial time.

Theorem 3.3 (Robust Stability Test): Suppose that C0, C1, C2 and Condition 3.1 hold and

where

$$
e_{0} \in \mathbb{E}, \quad\left[c_{0}^{\mathrm{d}}, \hat{d}_{0}\right] \in \mathbb{S}_{d}
$$

$$
\mathbb{S}_{d} \triangleq\left\{\left[c_{0}^{\mathrm{d}}, \hat{d}_{0}\right] \mid \exists \mu \text { such that }\left[c_{0}^{\mathrm{d}}, \mu\right] \in \mathbb{S}\left(\hat{d}_{0}\right)\right\}
$$

then Algorithm 3.1 robustly stabilizes the system (3).

Proof: See Appendix B.

\section{Robust Mixed ObJective MPC Design}

\section{A. Upper Bound on the Nominal Cost}

To compute the cost function matrix $P=P^{*}$, subject to Condition 3.1, as an "optimal" upper bound on the given nominal cost function matrix $P=P_{\left\{Q, R, R^{\mathrm{d}}\right\}}$ in (61), we adapt the procedure proposed in [13], [14] and consider the following SDP:

$$
\inf _{P, \Omega_{0}, m}\left\{\theta(P) \text { s.t. } \Sigma_{\left\{Q, R, R^{\mathrm{d}}\right\}}(P) \leq 0,(56) \text { and } m \geq 0\right\}
$$

$$
\begin{aligned}
& \text { where } \\
& \Sigma_{\left\{Q, R, R^{\mathrm{d}}\right\}}(P) \triangleq\left[\begin{array}{cc}
A_{0} & B_{I} D_{1} \\
0 & \Gamma
\end{array}\right]^{\mathrm{T}} P\left[\begin{array}{cc}
A_{0} & B_{I} D_{1} \\
0 & \Gamma
\end{array}\right]-P \\
& \quad+\left[\begin{array}{ccc}
I_{n_{x}} & 0 & 0 \\
0 & I_{n} & 0 \\
0 & -I_{n} & D_{1}
\end{array}\right]^{\mathrm{T}}\left[\begin{array}{ccc}
Q & 0 & 0 \\
0 & R & 0 \\
0 & 0 & R^{\mathrm{d}}
\end{array}\right]\left[\begin{array}{ccc}
I_{n_{x}} & 0 & 0 \\
0 & I_{n} & 0 \\
0 & -I_{n} & D_{1}
\end{array}\right]
\end{aligned}
$$

and where the criterion satisfies

$$
\begin{aligned}
\theta(P)= & (1-v) \operatorname{Trace}\left(W_{\left\{Q, R, R^{\mathrm{d}}\right\}}^{\mathrm{T}} P W_{\left\{Q, R, R^{\mathrm{d}}\right\}}\right) \\
& +v \operatorname{Trace}(P) \\
W_{\left\{Q, R, R^{\mathrm{d}}\right\}} \triangleq & {\left[\begin{array}{c}
I_{\left(n_{x}+n\right)} \\
-H_{0}^{-1} G_{0}
\end{array}\right],\left[\begin{array}{cc}
X_{0} & G_{0}^{\mathrm{T}} \\
G_{0} & H_{0}
\end{array}\right] \triangleq P_{\left\{Q, R, R^{\mathrm{d}}\right\}} }
\end{aligned}
$$

for a (small) selectable positive scalar $0<v \leq 1$.

Remark 4.1: We note that nominal cost function matrix $P_{\left\{Q, R, R^{\mathrm{d}}\right\}}$ in (61) satisfies

$$
\Sigma_{\left\{Q, R, R^{\mathrm{d}}\right\}}\left(P_{\left\{Q, R, R^{\mathrm{d}}\right\}}\right)=0 .
$$

Accordingly, any feasible solution, $P$, to (64) satisfies $P \geq$ $P_{\left\{Q, R, R^{\mathrm{d}}\right\}}$. The criterion (65) is motivated and studied in further detail in [13] and Section IV-C-2 below. As a general guideline, we suggest choosing the associated scalar $v$ to be a few orders of magnitude less than the singular values of the matrix $W_{\left\{Q, R, R^{\mathrm{d}}\right\}}$. In the numerical example below we use $v=10^{-5}$.

In the sequel, we use $P^{*}$ to denote a feasible and (near) optimal solution to (64). For nominal performance purposes, we would like the value chosen for $P^{*}$ to be a "tight" upper bound on $P_{\left\{Q, R, R^{\mathrm{d}}\right\}}$. Fortunately, as shown in the following technical result, this will always be the case when the model error is "sufficiently small".

Theorem 4.1: Suppose C1 holds. For any given $\epsilon>0$, there exists a $\delta>0$, such that, if we make the assignment $\left[G_{21}(z) G_{22}(z)\right] \leftarrow \delta\left[G_{21}(z) G_{22}(z)\right]$, then

$$
\operatorname{Trace}\left(P^{*}-P_{\left\{Q, R, R^{\mathrm{d}}\right\}}\right) \leq \epsilon \text {. }
$$

Proof: See the proof of Theorem 3.7 in [13].

Remark 4.2: Note that replacing $\left[G_{21}(z) G_{22}(z)\right]$ with $\left[\delta G_{21}(z) \delta G_{22}(z)\right]$ amounts to "shrinking" the uncertainty by a factor $\delta$ [see Remark 2.1], or, equivalently, scaling the matrix $C_{\mathrm{p}}$ in (60) to yield $C_{\mathrm{p}} \leftarrow \delta C_{\mathrm{p}}=\delta\left[C_{\mathrm{a}, z} D D_{1} 0\right]$.

\section{B. Proposed MPC Design}

By use of $P=P^{*}$ we obtain the following robust design procedure.

Algorithm 4.1: Off-line: (i) Determine the observer gain $L$, the integer $N_{\mathrm{s}}$ and the sets, $\mathbb{E}$ and $\mathcal{R}$, such that $\mathbf{C 0}, \mathbf{C 1}$ and $\mathbf{C 2}$ hold. (ii) Choose any integer $N \geq N_{\mathrm{s}}$. (iii) Choose weighting matrices $Q \geq 0, R \geq 0$ and $R^{\mathrm{d}}>0$ for the nominal cost function (61). (iv) Solve problem (64) to determine a (near) optimal and feasible $P^{*} \geq P_{\left\{Q, R, R^{\mathrm{d}}\right\}}$. Set $P=P^{*}$.

On-line: Implement Algorithm 3.1 using initial estimates such that (62) holds.

By straightforward application of previous results, we note that the proposed algorithm has the following key properties.

Theorem 4.2 (Properties of Algorithm 4.1): (Feasibility) If the first off-line (on-line) step of Algorithm 4.1 is feasible then the remaining off-line (on-line) steps are feasible.

(Robust Stability) Algorithm 4.1 robustly stabilizes the system (3). 
(Convergence to Nominal Cost) For any $\epsilon>0$, there exists a $\delta>0$, such that if the off-line steps of Algorithm 4.1 are carried out using $\left[G_{21}(z) G_{22}(z)\right] \leftarrow \delta\left[G_{21}(z) G_{22}(z)\right]$, then $\operatorname{Trace}\left(P^{*}-P_{\left\{Q, R, R^{\mathrm{d}}\right\}}\right) \leq \epsilon$.

\section{Numerical Example}

1) : We consider a discretized version of the "ill-conditioned" distillation column model employed in [30]. Specifically, we let the open-loop transfer function be of the form

$$
G_{\Delta}(z)=G_{11}(z)\left[I+W(z) \Delta_{\mathrm{T}}(z)\right]
$$

where $W(z)=0.8220(z-0.9018) /(z-0.0183)$, $\left\|\Delta_{T}(z)\right\|_{\infty} \leq 1$, and where the nominal model $G_{11}(z)$ is a zero-order hold discretizisation (with sampling time $T_{s}=4$ ) of

$$
G_{11}(s)=\frac{1}{75 s+1}\left[\begin{array}{cc}
87.8 & -86.4 \\
108.2 & -109.6
\end{array}\right] .
$$

Also, we consider unit bound input constraints and output disturbances described by use of $\mathbb{D}^{*}=\left\{d \in \mathbb{R}^{2} \|\left. d\right|_{\infty} \leq 0.25\right\}$ and $\mathbb{D}=\left\{d \in \mathbb{R}^{2} \|\left. d\right|_{\infty} \leq 0.05\right\}$ in Assumption 2.2.

To capture this system description, we choose $G(z)=$ $\left[\begin{array}{cc}G_{11}(z) & G_{11}(z) W(z) \\ I & 0\end{array}\right], C_{q}=0, D_{q}=I_{2}$ and $\mathbb{Q}=\mathbb{Z}=\mathbb{W}=\left\{u \in \mathbb{R}^{2} \|\left. u\right|_{\infty} \leq 1\right\}$. Note that Assumption 2.1 then holds and that, via our choice $\mathbb{W}=\mathbb{Q}$, we restrict attention to operators $\Delta$ which are such that $\left\{w_{k}\right\}=\Delta\left\{z_{k}\right\}=\Delta\left\{u_{k}\right\} \in \ell_{2 e}(\mathbb{Q})$. In (2), we choose the following minimal, balanced state space representation of $G(z)$ :

$$
\begin{aligned}
& A= \\
& {\left[\begin{array}{cccc}
9.4713 \cdot 10^{-1} & 8.7622 \cdot 10^{-2} & 0 & 0 \\
9.8975 \cdot 10^{-3} & 1.9249 \cdot 10^{-2} & 0 & 0 \\
0 & 0 & 9.4713 \cdot 10^{-1} & -8.7622 \cdot 10^{-2} \\
0 & 0 & -9.8975 \cdot 10^{-3} & 1.9249 \cdot 10^{-2}
\end{array}\right]} \\
& B=\left[\begin{array}{cc}
-2.2595 & 2.2631 \\
-2.4077 \cdot 10^{-2} & 2.4115 \cdot 10^{-2} \\
-1.9009 \cdot 10^{-1} & -1.8979 \cdot 10^{-1} \\
2.0256 \cdot 10^{-3} & 2.0224 \cdot 10^{-3}
\end{array}\right] \\
& B_{w}=\left[\begin{array}{cc}
-2.7564 \cdot 10^{-1} & 2.7607 \cdot 10^{-1} \\
1.9418 & -1.9449 \\
-2.3189 \cdot 10^{-2} & -2.3153 \cdot 10^{-2} \\
-1.6336 \cdot 10^{-1} & -1.6311 \cdot 10^{-1}
\end{array}\right] \\
& C=\left[\begin{array}{cccc}
-2.0178 & 1.6271 & -2.1191 \cdot 10^{-1} & -1.7088 \cdot 10^{-1} \\
-2.5229 & 2.0343 & 1.6949 \cdot 10^{-1} & 1.3667 \cdot 10^{-1}
\end{array}\right] \\
& C_{z}=0, \quad D=I \text {. }
\end{aligned}
$$

For our estimator design we choose

$$
L=\left[\begin{array}{cc}
-5.2485 \cdot 10^{-3} & -6.5623 \cdot 10^{-3} \\
3.4872 \cdot 10^{-3} & 4.3600 \cdot 10^{-3} \\
-1.3538 \cdot 10^{-4} & 1.0828 \cdot 10^{-4} \\
-4.7326 \cdot 10^{-4} & 3.7852 \cdot 10^{-4} \\
6.3330 \cdot 10^{-1} & -4.4801 \cdot 10^{-1} \\
-4.4801 \cdot 10^{-1} & 4.3147 \cdot 10^{-1}
\end{array}\right]
$$

and

$$
\mathbb{E}=\mathbb{F}(\alpha, s), \quad \alpha=0.313, \quad s=400
$$

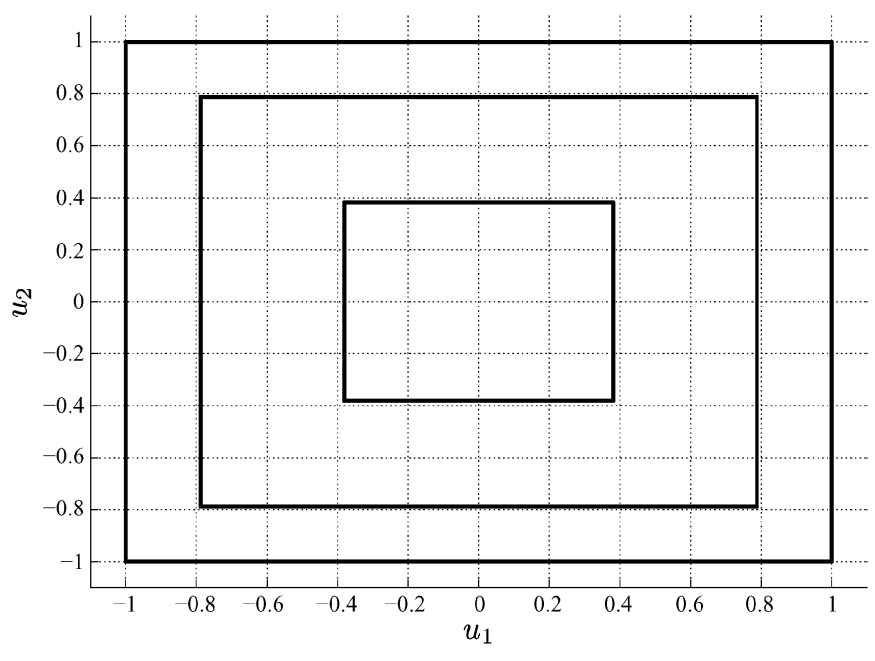

Fig. 2. Sets $\mathbb{Q}=\hat{\mathbb{Q}}_{0} \supset \hat{\mathbb{Q}}_{1} \supset \hat{\mathbb{Q}}_{1}^{\mathrm{d}}$ in $\mathbf{C} 2$ when $N_{\mathrm{s}}=2$ and $\mathbb{E}$ is as in (68).

where ${ }^{3}$

$$
\begin{aligned}
\mathbb{F}(\alpha, s) & =(1-\alpha)^{-1}\left(\mathcal{W} \oplus A_{L} \mathcal{W} \oplus \cdots \oplus A_{L}^{(s-1)} \mathcal{W}\right) \\
\mathcal{W} & =\left\{\left[\begin{array}{cc}
B_{w} & -L_{1} \\
0 & -L_{2}
\end{array}\right] \mathbb{W} \times \mathbb{D}\right\} \oplus\left\{\omega \mid\|\omega\|_{\infty} \leq 10^{-5}\right\} .
\end{aligned}
$$

That is, following [31], we take the set $\mathbb{E}$ to be an invariant approximation of the minimal robustly invariant set for the system $e_{i+1}=A_{L} e_{i}+\omega_{i}$ with input $\omega_{i} \in \mathcal{W}$. Condition $\mathbf{C 0}$ thus holds. Moreover, since $\left\|C_{\mathrm{a}, z}\left(z I-A_{\mathrm{a}}\right)^{-1} B_{\mathrm{a}, w}\right\|_{\infty}=0.0822$, it follows that $\mathbf{C 1}$ also holds. Next, to satisfy $\mathbf{C 2}$, we consider $N_{\mathrm{s}}=2$ and we then obtain the sets, $\hat{\mathbb{Q}}_{0}, \hat{\mathbb{Q}}_{1}, \hat{\mathbb{Q}}_{1}^{\mathrm{d}}$, shown in Fig. 2. Hence, since we only have input constraints (i.e., $C_{q}=0$ ) and $0 \in \hat{\mathbb{Q}}_{1}^{\mathrm{d}}$, we choose $\mathcal{R}=\mathbb{R}^{6}$ (and $N_{\mathrm{s}}=2$ ) to satisfy $\mathbf{C 2}$.

In Algorithm 4.1, we choose $N=10, Q=C^{\mathrm{T}} C$ and $R=R^{\mathrm{d}}=10^{-4} I$. Nominal responses of the resulting instance of Algorithm 4.1, obtained when $e_{0}=0,\left\{d_{k}\right\}_{k=0}^{\infty}=0, \Delta=0$ and $d^{*}=[-0.25,0.25] \in \mathbb{D}^{*}$, are shown in Fig. 3. For comparison purposes, Fig. 3 also shows: (i) the associated open-loop response (obtained when $\left\{u_{k}\right\}_{k=0}^{\infty}=0$ ); and (ii) the closed-loop response of an associated "nominal" MPC (NOMPC) design, namely, Algorithm 4.1 but using the nominal cost function matrix $P=P_{\left\{Q, R, R^{\mathrm{d}}\right\}}$, rather than the associated upper bound $P^{*}$. It can be seen that both Algorithm 4.1 and the NOMPC design significantly improve on the open-loop system response, and remove the steady-state error caused by the output disturbance $d^{*}$. We also note that Algorithm 4.1 seems only to be slightly conservative as compared with the NOMPC design. Moreover, unlike Algorithm 4.1, the NOMPC design is not robustly stable, as illustrated next. In Fig. 4 the simulation of Fig. 3 is repeated using non-zero, admissible realizations of $e_{0},\left\{d_{k}\right\}_{k=0}^{\infty}$ and $\Delta$, namely, $e_{0} \in \mathbb{E},\left\{d_{k}\right\}_{k=0}^{\infty}=\left\{0.05 \exp ^{-(k / 10)}\right\}_{k=0}^{\infty} \in \ell_{2}(\mathbb{D})$ and $\Delta \in \Delta^{+}(\mathbb{Z}, \mathbb{W})$ described by $\mathbf{w}^{0}=0$ and $\Delta_{\mathrm{T}}(z)=$ $\operatorname{diag}\left\{1, z^{-3}\right\}$ [see (1)]. Hence, it follows by Theorem 3.3 that Algorithm 4.1 will be closed-loop stable. On the other hand, the NOMPC design, which uses the nominal cost, fails severely for

${ }^{3}$ In (70), we have added a small hypercube so that the resulting set $\mathcal{W}$ contains the origin in its interior (c.f. [31]). 

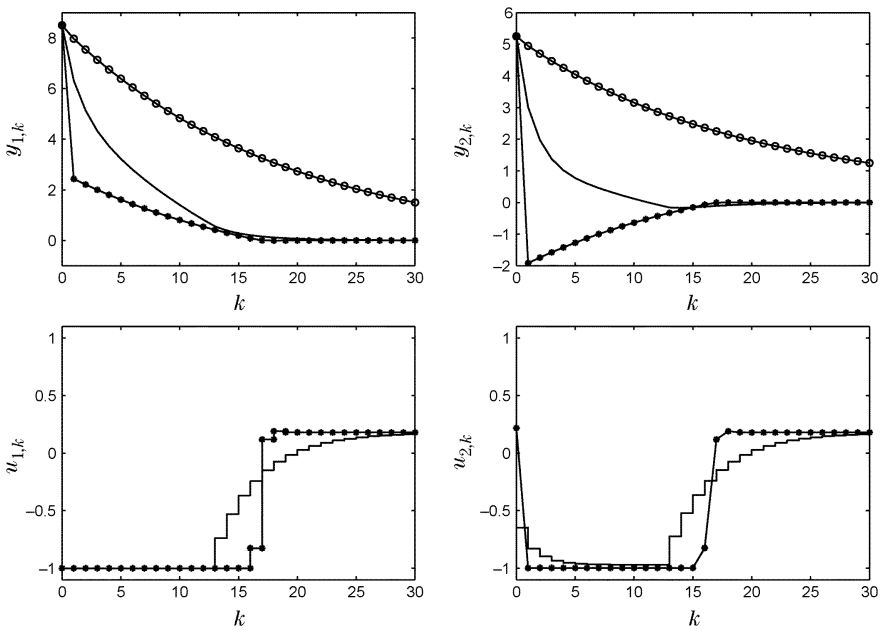

Fig. 3. Nominal responses: Open-loop response (o-solid) and closed-loop responses, $y_{k}=\left[y_{1, k}, y_{2, k}\right]$ and $u_{k}=\left[u_{1, k}, u_{2, k}\right]$, of NOMPC (•-solid) and Algorithm 4.1 (solid), when $x_{0}=[-2.22,-10.6,0,0]$.
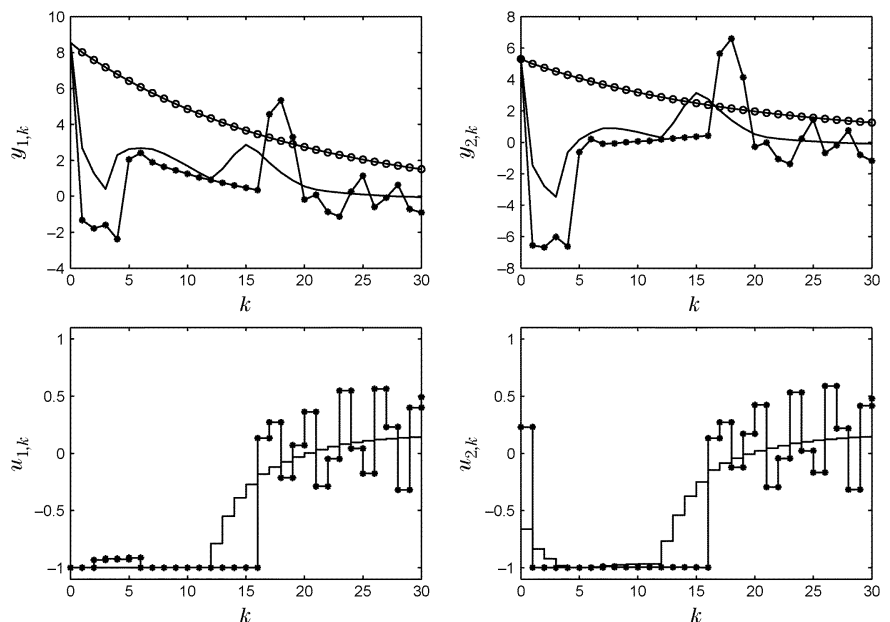

Fig. 4. Perturbed responses: Open-loop response (o-solid) and closed-loop responses, $y_{k}=\left[y_{1, k}, y_{2, k}\right]$ and $u_{k}=\left[u_{1, k}, u_{2, k}\right]$, of NOMPC (•-solid) and Algorithm 4.1 (solid), when $x_{0}=[-2.22,-10.6,0,0]$.

this particular admissible realization of $\Delta$. In particular, the control inputs become oscillatory. From simulations over a longer time interval, we note that these oscillations eventually converge to a periodic motion which frequently hits the input constraints.

2) : To illustrate Theorem 4.1, we next repeat the cost function computations of the preceding example using different values for $C_{p}$. Specifically, we consider $C_{p} \leftarrow \delta C_{p}$ for a set of $\delta \in(0,1]$ [see Remark 4.2]. (When $\delta=1$ we recover the robust design in Figs. 3 and 4). Let $P^{\delta}=P^{*}-P_{\left\{Q, R, R^{\mathrm{d}}\right\}}$ denote the "added weighting" obtained for a particular $\delta$. Theorem 4.1 shows that (the trace of) $P^{\delta} \geq 0$ goes to zero as $\delta$ goes to zero. This is confirmed by the top plot in Fig. 5 .

Given a particular $\delta$, let $K^{\delta}\left(P^{*}\right)$ denote the underlying feedback gain of Algorithm 4.1, and let $\Lambda_{\left\{P^{*}\right\}}^{\delta}(z)$ denote the closedloop transfer function associated with $K^{\delta}\left(P^{*}\right)$ and the LQR problem with cost (61), that is

$$
\begin{aligned}
\Lambda_{\{P\}}^{\delta}(z) \triangleq & {\left[\begin{array}{c}
\operatorname{diag}\{Q, R\}^{1 / 2}\left(z I-A_{0}+B_{I} K^{\delta}(P)\right)^{-1} \\
-\left(R^{\mathrm{d}}\right)^{1 / 2} K^{\delta}(P)\left(z I-A_{0}+B_{I} K^{\delta}(P)\right)^{-1}
\end{array}\right], } \\
K^{\delta}(P) & =K^{\delta}\left(\left[\begin{array}{cc}
X & G^{\mathrm{T}} \\
G & H
\end{array}\right]\right) \triangleq D_{1} H^{-1} G .
\end{aligned}
$$
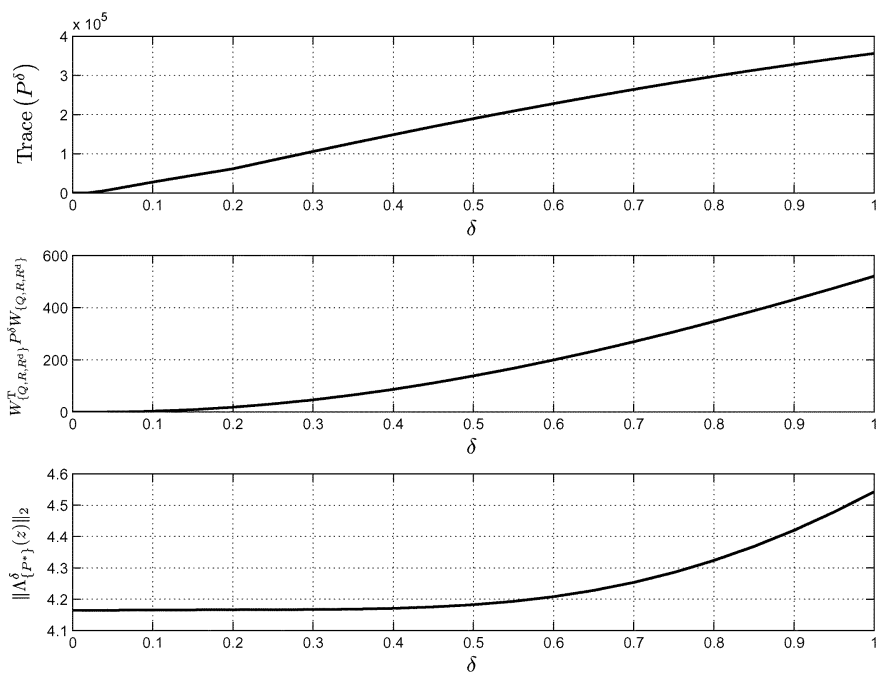

Fig. 5. $\left\|\Lambda_{\left\{P^{*}\right\}}^{\delta}(z)\right\|_{2}$ increases (slowly) as the model uncertainty, parameterized by $\delta$, increases.

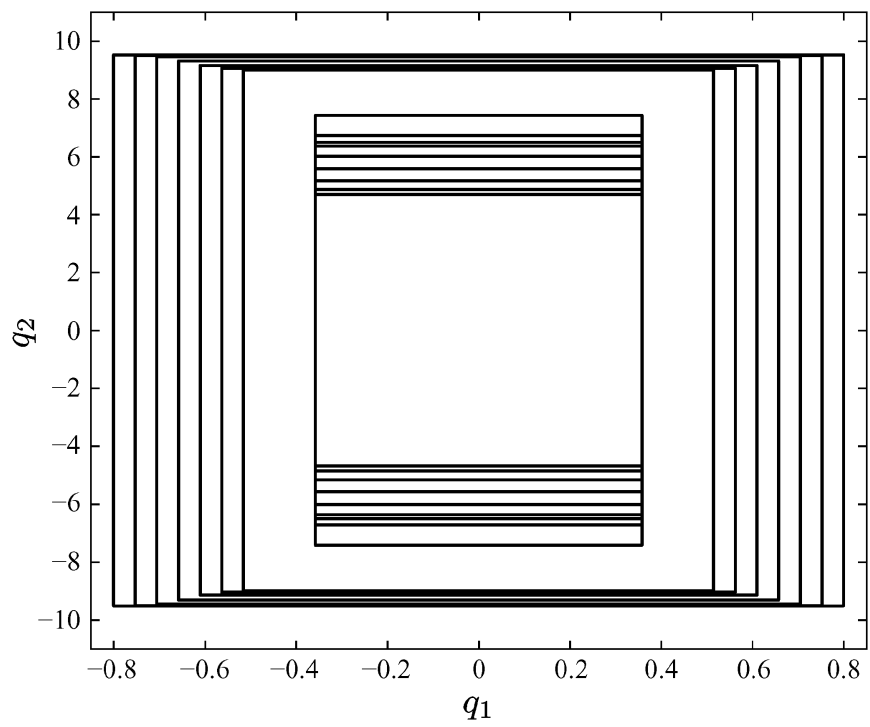

Fig. 6 Sets $\hat{\mathbb{Q}}_{0} \supset \hat{\mathbb{Q}}_{1} \supset \cdots \supset \hat{\mathbb{Q}}_{7} \supset \hat{\mathbb{Q}}_{7}^{\mathrm{d}} \supset \cdots \supset \hat{\mathbb{Q}}_{15}^{\mathrm{d}}$ in C2 when $N_{\mathrm{s}}=7$ and $N=15$.

The bottom plot in Fig. 5 shows how the $H_{2}$ norm of $\Lambda_{\left\{P^{*}\right\}}^{\delta}(z)$ evolves with $\delta$. Note that practically no increase occurs on the interval $\delta \in(0,0.4] .{ }^{4}$ Also note from Fig. 5 that we actually have $\operatorname{Trace}\left(P^{0.1}\right) \approx 2.8 \cdot 10^{4}$. Hence, the added weighting $P^{\delta}=P^{*}-P_{\left\{Q, R, R^{\mathrm{d}}\right\}}$ incorporated in Algorithm 4.1 may be rather large and still result in excellent nominal performance with respect to the given weighting matrices $Q$, $R, R^{\mathrm{d}}$. This very important property is made possible by the fact that the added weighting may be associated with directions in the solution space of the optimization problem that are orthogonal to the nominal solution. As an example, we note that although $\operatorname{Trace}\left(P^{0.1}\right) \approx 2.8 \cdot 10^{4}$ we actually have $\operatorname{Trace}\left(W_{\left\{Q, R, R^{\mathrm{d}}\right\}}^{\mathrm{T}} P^{0.1} W_{\left\{Q, R, R^{\mathrm{d}}\right\}}\right) \approx 3.0$ [see (66)]. Thus the added weighting is almost ortoghonal to the rows of the matrix $W_{\left\{Q, R, R^{\mathrm{d}}\right\}}$. Also note from Fig. 5 that the feedback gain

${ }^{4}$ The $H_{2}$ norm of a transfer function $\Lambda(z)$ is given by $\|\Lambda(z)\|_{2}=$ $\left((1 / 2 \pi) \int_{-\pi}^{\pi} \text { Trace }\left[\Lambda^{*}\left(\mathrm{e}^{\mathrm{j} \omega}\right) \Lambda\left(\mathrm{e}^{\mathrm{j} \omega}\right)\right] d \omega\right)^{1 / 2}$. 

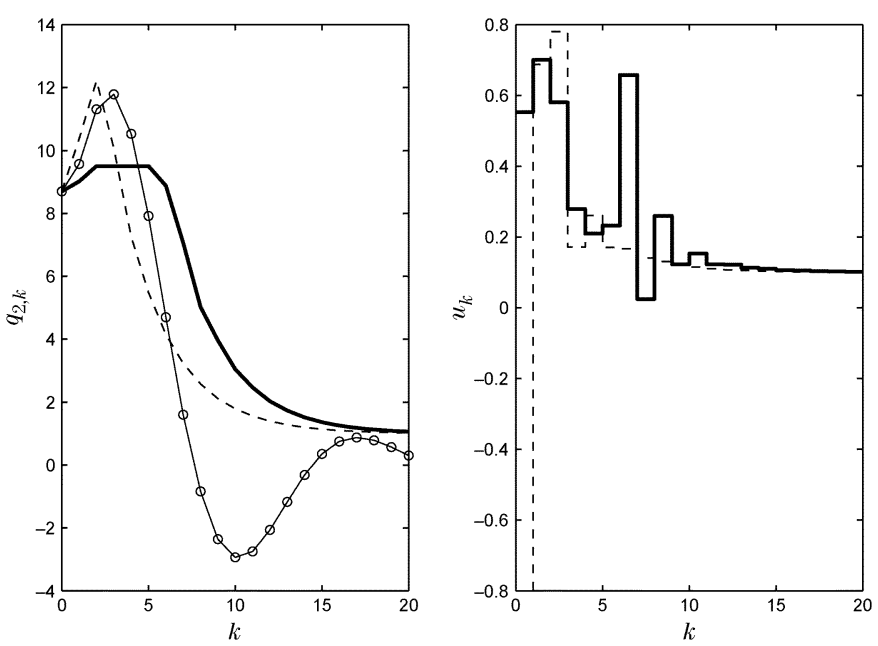

Fig. 7 Open-loop response (o-solid) and closed-loop responses of Algorithm 4.1 with (solid) and without (dashed) the constraint $\left|q_{2, k}\right| \leq 10$.

corresponding to the robust design in Figs. 3, 4 achieves an $\mathrm{H}_{2}$ norm of 4.54 which is only 0.38 above the optimum of 4.16 , and far less than the $\mathrm{H}_{2}$ norm of the "open-loop dynamics", that is, $\left\|\operatorname{diag}\{Q, R\}^{1 / 2}\left(z I-A_{0}\right)^{-1}\right\|_{2}=10.47$.

$3)$ : To illustrate the output constraint handling capabilities of the proposed design, we consider an uncertain version of the non-minimum phase system studied in [32], that is

$$
\begin{aligned}
& A=\left[\begin{array}{cccc}
0.9234 & 0.3562 & 0.0227 & 0.0026 \\
-0.3561 & 0.7505 & 0.2914 & -0.0059 \\
0.0228 & -0.2912 & 0.3267 & 0.1464 \\
0.0003 & 0.0017 & -0.0002 & 0.2494
\end{array}\right] \\
& B=\left[\begin{array}{c}
-0.7143 \\
-1.8571 \\
-1.3917 \\
0.0047
\end{array}\right] \\
& C=\left[\begin{array}{llll}
-0.7152 & 1.8571 & -1.3922 & 0.1012
\end{array}\right] \text {, } \\
& B_{w}=\left[\begin{array}{c}
-0.0276 \\
-0.0202 \\
0.0340 \\
0.2887
\end{array}\right] \\
& C_{z}=0, \quad D=1, \quad C_{q}=\left[\begin{array}{ll}
0 & 0 \\
C & 0
\end{array}\right], \quad D_{q}=\left[\begin{array}{l}
1 \\
0
\end{array}\right]
\end{aligned}
$$

with $\mathbb{Z}=\mathbb{W}=\{u \in \mathbb{R}|| u \mid \leq 0.8\}, \mathbb{Q}=\left\{\left[q_{1}, q_{2}\right] \in\right.$ $\left.\mathbb{R}^{2}|| q_{1}|\leq 0.8,| q_{2} \mid \leq 10\right\}$ and $\mathbb{D}^{*}=\{d \in \mathbb{R} \| d \mid \leq 1\}$ and $\mathbb{D}=\{d \in \mathbb{R}|| d \mid \leq 0.1\}$ in Assumption 2.2. For the observer gain we consider a Kalman filter design given by $L=[-0.0018,0.0045,-0.0020,-0.0020,0.9102]$. We note that this leads to satisfaction of C1, since $\left\|C_{\mathrm{a}, z}\left(z I-A_{\mathrm{a}}\right)^{-1} B_{\mathrm{a}, w}\right\|_{\infty}=0.0409$. As above, we take the associated set in $\mathbf{C O}$ to be an invariant approximation of the minimal choice for $\mathbb{E}$, namely $\mathbb{E}=\mathbb{F}(\alpha, s)$ with $\alpha=8.62 \cdot 10^{-4}$ and $s=100$ [see (68)-(70)]. To satisfy C2 we use Algorithm 6.2 in [28] to compute an invariant for the system in (31), where we take $N_{\mathrm{s}}=7$. This results in a set $\mathcal{R}$ described by 12 linear inequalities. The associated sequence of "output sets" are shown in Fig. 6. In Algorithm 4.1, we choose
$N=15, Q=C^{\mathrm{T}} C, R=0.1$ and $R^{\mathrm{d}}=0.01$. Closed-loop responses of the resulting instance of Algorithm 4.1, obtained when $e_{0}=0,\left\{d_{k}\right\}_{k=0}^{\infty}=0, \Delta=0$ and $d^{*}=-1 \in \mathbb{D}^{*}$, are shown in Fig. 7. To illustrate the trade-off between performance and constraint satisfaction, Fig. 7 also shows the closed-loop responses of another instance of Algorithm 4.1 obtained by omitting the output constraint (i.e., by considering $\left.\mathbb{Q}=\left\{\left[q_{1}, q_{2}\right] \in \mathbb{R}^{2} \|\left. q_{1}\right|_{\infty} \leq 0.8\right\}\right)$.

\section{CONCLUSION}

We have proposed a robust output-feedback MPC design for a class of square, open-loop stable systems, having non-vanishing output disturbances, hard constraints and linear-time invariant model uncertainty. The design incorporates a novel closed-loop stability test and minimizes a quadratic upper bound on a nominal cost function at each time step. An important distinction relative to previous work is that we have proven robust convergence to the correct steady-state in the face of both imperfect state information and dynamic model uncertainty. As shown in Appendix $\mathrm{C}$, the proposed design can easily be generalized to a class of open-loop unstable systems by means of a pre-stabilization technique (i.e., by changing the candidate policy). On the other hand, it seems to be a challenging topic for future work to generalize our results to incorporate different disturbance models and non-square systems.

\section{APPENDIX A}

The MATRICES $\Lambda_{1}, \Lambda_{2}, \Lambda_{3}, G_{1}, G_{2}$

The dynamics of $e_{k}^{\mathrm{d}}$ in (23) follow from (15). To verify the dynamics of $c_{k}^{\mathrm{d}}$ in (23), consider

$$
\begin{aligned}
c_{k+1}^{\mathrm{d}}= & c_{k+1}-K_{1} \hat{d}_{k+1} \\
= & A_{0} c_{k}-B_{I}\left[C(I-A)^{-1} B\right]^{-1} \hat{d}_{k}+B_{I} v_{k}^{*} \\
& +\left[\begin{array}{c}
L_{1} \\
0
\end{array}\right]\left(\left[\begin{array}{ll}
C & I
\end{array}\right] e_{k}+d_{k}\right) \\
& -K_{1} \hat{d}_{k}-K_{1} L_{2}\left(\left[\begin{array}{ll}
C & I
\end{array}\right] e_{k}+d_{k}\right) \\
= & A_{0} c_{k}-A_{0} K_{1} \hat{d}_{k}+B_{I} v_{k}^{*}+\Lambda_{1} e_{k}+\Lambda_{2} d_{k} \\
= & A_{0} c_{k}^{\mathrm{d}}+B_{I} v_{k}^{*}+\Lambda_{1} e_{k}^{\mathrm{d}}+\Lambda_{2} d_{k}
\end{aligned}
$$

where we have made use of (3), (9), (11), (16)-(18), (25), the identity

$$
A_{0} K_{1}=K_{1}+B_{I}\left[C(I-A)^{-1} B\right]^{-1}
$$

and the fact that $\Lambda_{1} \mathrm{e}^{*}(\Delta)=0$ so that $\Lambda_{1} e_{k}^{\mathrm{d}}=\Lambda_{1} e_{k}$ (c.f. (13), (14) and (25)).

The matrices $G_{1}$ and $G_{2}$ in (28), (29) are defined to be such that

$$
\begin{aligned}
{\left[x_{k}-\hat{x}_{k}\right]+\left[\hat{x}_{k}^{*}-\mathbf{x}^{*}(\Delta)\right] } & =G_{1} e_{k}^{\mathrm{d}} \\
\hat{u}_{k}^{*}-\mathbf{u}^{*}(\Delta) & =G_{2} e_{k}^{\mathrm{d}} .
\end{aligned}
$$

To verify this, we make use of (13), (14) and the identity

$\mathbf{u}^{*}(\Delta)=-\left[C(I-A)^{-1} B\right]^{-1} \times\left(d^{*}+C(I-A)^{-1} B_{w} \mathbf{w}^{*}(\Delta)\right)$ 
(which follows from $C \mathbf{x}^{*}(\Delta)=-d^{*}$ ) and consider:

$$
\begin{aligned}
{\left[x_{k}-\right.} & \left.\hat{x}_{k}\right]+\left[\hat{x}_{k}^{*}-\mathbf{x}^{*}(\Delta)\right] \\
= & x_{k}-\hat{x}_{k}+(I-A)^{-1} B \hat{u}_{k}^{*} \\
& -(I-A)^{-1} B_{w} \mathbf{w}^{*}(\Delta)-(I-A)^{-1} B \mathbf{u}^{*}(\Delta) \\
= & {[I \quad 0] e_{k}^{\mathrm{d}}-(I-A)^{-1} B \mathbf{u}^{*}(\Delta) } \\
& -(I-A)^{-1} B\left[C(I-A)^{-1} B\right]^{-1} \hat{d}_{k} \\
= & {[I \quad 0] e_{k}^{\mathrm{d}}+(I-A)^{-1} B\left[C(I-A)^{-1} B\right]^{-1} } \\
& \times\left(d^{*}-\hat{d}_{k}+C(I-A)^{-1} B_{w} \mathbf{w}^{*}(\Delta)\right) \\
= & G_{1} e_{k}^{\mathrm{d}}
\end{aligned}
$$

and

$$
\begin{aligned}
& \hat{u}_{k}^{*}-\mathbf{u}^{*}(\Delta) \\
& =\left[C(I-A)^{-1} B\right]^{-1}\left(d^{*}-\hat{d}_{k}+C(I-A)^{-1} B_{w} \mathbf{w}^{*}(\Delta)\right) \\
& =G_{2} e_{k}^{\mathrm{d}} .
\end{aligned}
$$

It remains to verify (23b). This can be done by using (19) and (75) as follows:

$$
\begin{aligned}
z_{k}^{\mathrm{d}} & =C_{z}\left(x_{k}-\mathbf{x}^{*}(\Delta)\right)+D\left(u_{k}-\mathbf{u}^{*}(\Delta)\right) \\
& =C_{z}\left(G_{1} e_{k}^{\mathrm{d}}+\hat{x}_{k}-\hat{x}_{k}^{*}\right)+D\left(G_{2} e_{k}^{\mathrm{d}}+v_{k}^{*}\right) \\
& =\Lambda_{3} e_{k}^{\mathrm{d}}+\left[\begin{array}{ll}
C_{z} & 0
\end{array}\right] c_{k}^{\mathrm{d}}+D v_{k}^{*}
\end{aligned}
$$

\section{APPENDIX B}

\section{PROOF OF THEOREM 3.3}

To prove Theorem 3.3 we will need the following two lemmas which relate $\left\{w_{k}^{\mathrm{d}}\right\}_{k=0}^{\infty}$ to $\left\{z_{k}^{\mathrm{d}}\right\}_{k=0}^{\infty}$.

Lemma B.1: Consider any $\Delta \in \boldsymbol{\Delta}^{+}(\mathbb{Z}, \mathbb{W})$ and any realization of $\left\{u_{k}\right\}_{k=0}^{\infty} \in \ell_{2 e}$ such that $\left\{q_{k}\right\}_{k=0}^{\infty} \in \ell_{2 e}(\mathbb{Q})$. For some transfer function $\Delta_{\mathrm{T}}(z) \in \Delta_{\mathrm{ESLTI}}$, satisfying $\left\|\Delta_{\mathrm{T}}(z)\right\|_{\infty} \leq 1$, and some sequence $\mathbf{c} \in \ell_{2}$, we have

$$
\left\{w_{k}^{\mathrm{d}}\right\}_{k=0}^{\infty}=\Delta_{\mathrm{T}}(z)\left\{z_{k}^{\mathrm{d}}\right\}_{k=0}^{\infty}+\mathbf{c} .
$$

Proof: By definition

$$
\begin{aligned}
\left\{w_{k}^{\mathrm{d}}\right\}_{k=0}^{\infty}= & \left\{w_{k}-\mathbf{w}^{*}(\Delta)\right\}_{k=0}^{\infty} \\
= & \Delta_{\mathrm{T}}(z)\left\{z_{k}\right\}_{k=0}^{\infty}+\mathbf{w}^{0}-\left\{\mathbf{w}^{*}(\Delta), \mathbf{w}^{*}(\Delta), \cdots\right\} \\
= & \Delta_{\mathrm{T}}(z)\left\{z_{k}^{\mathrm{d}}+\mathbf{z}^{*}(\Delta)\right\}_{k=0}^{\infty}+\mathbf{w}^{0} \\
& -\left\{\Delta_{\mathrm{T}}(1) \mathbf{z}^{*}(\Delta), \Delta_{\mathrm{T}}(1) \mathbf{z}^{*}(\Delta), \cdots\right\} \\
= & \Delta_{\mathrm{T}}(z)\left\{z_{k}^{\mathrm{d}}\right\}_{k=0}^{\infty}+\mathbf{w}^{0}+\mathbf{e}
\end{aligned}
$$

where

$$
\mathbf{e} \triangleq\left\{e_{k}\right\}_{k=0}^{\infty}, \quad e_{k} \triangleq\left[\sum_{n=k+1}^{\infty} h_{n}\right] \mathbf{z}^{*}(\Delta)
$$

and where $\Delta_{\mathrm{T}}(z)=\sum_{k=0}^{\infty} h_{k} z^{-k}$. Since $\mathbf{w}^{0} \in \ell_{2}$, it only remains to show that $\mathbf{e}$ is also in $\ell_{2}$. To this end, note that, since $\Delta_{\mathrm{T}}(z) \in \Delta_{\mathrm{ESLTI}}$, we have

$$
\begin{aligned}
\left\|e_{k}\right\| & \leq\left[\sum_{n=k+1}^{\infty}\left\|h_{k}\right\|_{2}\right]\left\|\mathbf{z}^{*}(\Delta)\right\| \\
& \leq\left[\sum_{n=k+1}^{\infty} a^{k}\right]\left\|\mathbf{z}^{*}(\Delta)\right\|=a^{k} \frac{a}{1-a}\left\|\mathbf{z}^{*}(\Delta)\right\|
\end{aligned}
$$

for some scalar $0 \leq a<1$. Hence

$$
\begin{aligned}
\sum_{k=0}^{\infty}\left\|e_{k}\right\|^{2} & \leq \sum_{k=0}^{\infty} a^{2 k}\left(\frac{a}{1-a}\right)^{2}\left\|\mathbf{z}^{*}(\Delta)\right\|^{2} \\
& \leq \sum_{k=0}^{\infty} a^{k}\left(\frac{a}{1-a}\right)^{2}\left\|\mathbf{z}^{*}(\Delta)\right\|^{2} \\
& =\frac{a^{2}}{(1-a)^{3}}\left\|\mathbf{z}^{*}(\Delta)\right\|^{2}<\infty .
\end{aligned}
$$

Lemma B.2: Let $\gamma$ be any scalar satisfying $\gamma>1$, and consider any $\Delta \in \Delta^{+}(\mathbb{Z}, \mathbb{W})$ and any realization of $\left\{u_{k}\right\}_{k=0}^{\infty} \in \ell_{2 e}$ such that $\left\{q_{k}\right\}_{k=0}^{\infty} \in \ell_{2 e}(\mathbb{Q})$. For some scalar $c \geq 0$ and all $L \in \mathbb{N}^{+}$, we have

$$
\sum_{k=0}^{L}\left\|w_{k}^{\mathrm{d}}\right\|^{2} \leq c+\gamma^{2} \sum_{k=0}^{L}\left\|z_{k}^{\mathrm{d}}\right\|^{2} .
$$

Proof: Let $\mathbf{P}_{L}$ denote the usual (time) truncation operator, that is

$$
\mathbf{P}_{L}\left\{z_{k}\right\}_{0}^{\infty}=\left\{\left\{z_{k}\right\}_{k=0}^{L}, 0,0, \cdots\right\} .
$$

By Lemma B.1 and causality, we have, for any $L \in \mathbb{N}^{+}$

$$
\sum_{k=0}^{L}\left\|w_{k}^{\mathrm{d}}\right\|^{2} \leq\left\|\Delta_{\mathrm{T}}(z) \mathbf{P}_{L}\left\{z_{k}^{\mathrm{d}}\right\}_{k=0}^{\infty}+\mathbf{P}_{L} \mathbf{c}\right\|^{2} .
$$

To proceed will use the fact that, since $\left\|\Delta_{\mathrm{T}}(z)\right\|_{\infty}<\gamma$, there exists $\alpha>0$ such that $\left\|\Delta_{\mathrm{T}}(z)\right\|_{\infty} \leq \gamma / \sqrt{1+\alpha}$. Hence

$$
\begin{aligned}
\sum_{k=0}^{L}\left\|w_{k}^{\mathrm{d}}\right\|^{2} \leq & \left\|\Delta_{\mathrm{T}}(z) \mathbf{P}_{L}\left\{z_{k}^{\mathrm{d}}\right\}_{k=0}^{\infty}+\mathbf{P}_{L} \mathbf{c}\right\|^{2} \\
\leq & (1+\alpha)\left\|\Delta_{\mathrm{T}}(z) \mathbf{P}_{L}\left\{z_{k}^{\mathrm{d}}\right\}_{k=0}^{\infty}\right\|^{2} \\
& +\left(1+\alpha^{-1}\right)\left\|\mathbf{P}_{L} \mathbf{c}\right\|^{2} \\
\leq & (1+\alpha) \frac{\gamma^{2}}{1+\alpha}\left\|\mathbf{P}_{L}\left\{z_{k}^{\mathrm{d}}\right\}_{k=0}^{\infty}\right\|^{2} \\
& +\left(1+\alpha^{-1}\right)\|\mathbf{c}\|^{2} \\
= & c+\gamma^{2} \sum_{k=0}^{L}\left\|z_{k}^{\mathrm{d}}\right\|^{2}
\end{aligned}
$$

where $c \triangleq\left(1+\alpha^{-1}\right)\|\mathbf{c}\|^{2}$. Note that the second inequality follows by the basic inequality $\|x+y\|^{2} \leq(1+\alpha) \|\left. x\right|^{2}+$ $\left(1+\alpha^{-1}\right)\|y\|^{2}$ [which holds for any $\alpha>0$ since $\|x+y\|^{2} \leq$ $\|x\|^{2}+2\|x\|\|y\|+\|y\|^{2}=-\left(\alpha^{1 / 2}\|x\|-\alpha^{-1 / 2}\|y\|\right)^{2}+(1+$ $\alpha)\|x\|^{2}+\left(1+\alpha^{-1}\right)\|y\|^{2}$ ]. The third inequality follows from 
$\left\|\Delta_{\mathrm{T}}(z)\right\|_{\infty} \leq \gamma / \sqrt{1+\alpha}$. This completes the proof since $c$ does not depend on $L$.

We are now in a position to prove Theorem 3.3.

Proof: As a first step, note from Theorem 3.1 that the set $\mathbb{E} \times \mathbb{S}_{d}$ is invariant for the closed-loop system, that is

$$
\left[e_{k}, c_{k}^{\mathrm{d}}, \hat{d}_{k}\right] \in \mathbb{E} \times \mathbb{S}_{d}, \quad \forall k \in \mathbb{N}^{+} .
$$

Hence, robust constraint satisfaction follows from (34) and (41), that is, (101) implies $\left\{q_{k}\right\}_{k=0}^{\infty} \in \ell_{2 e}(\mathbb{Q})$. To prove that both $\left\{x_{k}-\mathbf{x}^{*}(\Delta)\right\}_{k=0}^{\infty}$ and $\left\{u_{k}-\mathbf{u}^{*}(\Delta)\right\}_{k=0}^{\infty}$ are in $\ell_{2}$ (i.e., (8)), for any admissible uncertainty $\Delta$, consider the "storage function" candidate

$$
\begin{aligned}
V\left(e_{k}^{\mathrm{d}}, c_{k}^{\mathrm{d}}, \hat{d}_{k}\right) & \triangleq\left\|\left[e_{k}^{\mathrm{d}}, c_{k}^{\mathrm{d}}, K\left(\left[c_{k}^{\mathrm{d}}, \hat{d}_{k}\right]\right)\right]\right\|_{\boldsymbol{\Omega}\left(\Omega_{0}, P\right)}^{2} \\
& =\left\|\left[e_{k}^{\mathrm{d}}, c_{k}^{\mathrm{d}}\right]\right\|_{\Omega_{0}}^{2}+J^{*}\left(c_{k}^{\mathrm{d}}, \hat{d}_{k}\right)
\end{aligned}
$$

where

$$
\begin{aligned}
J^{*}\left(c_{k}^{\mathrm{d}}, \hat{d}_{k}\right) & \triangleq J\left(c_{k}^{\mathrm{d}}, K\left(\left[c_{k}^{\mathrm{d}}, \hat{d}_{k}\right]\right)\right) \\
& =\left\|\left[c_{k}^{\mathrm{d}}, K\left(\left[c_{k}^{\mathrm{d}}, \hat{d}_{k}\right]\right)\right]\right\|_{P}^{2} .
\end{aligned}
$$

Note from (56) that the function $V\left(e_{k}^{\mathrm{d}}, c_{k}^{\mathrm{d}}, \hat{d}_{k}\right)$ is non-negative and from (63) that it is well-defined for all $\left[e_{k}^{\mathrm{d}}, c_{k}^{\mathrm{d}}, \hat{d}_{k}\right] \in$ $\mathbb{R}^{\left(n_{x}+n\right)} \times \mathbb{S}_{d}$. Also note that, whenever (56) holds, there exists a scalar $\gamma>1$ and a sufficiently large scalar $\eta>0$ such that

$$
\Phi\left(\Omega_{0}, P, m, \gamma, \eta\right)<0
$$

where

$$
\begin{aligned}
\boldsymbol{\Phi}\left(\Omega_{0}, P, m, \gamma, \eta\right) \triangleq & {\left[\begin{array}{ccc}
A_{\mathrm{p}} & B_{\mathrm{p}} & \left.\left[\begin{array}{c}
B_{\mathrm{a}, d} \\
0
\end{array}\right]\right]^{\mathrm{T}} \\
\gamma C_{\mathrm{p}} & 0 & 0
\end{array}\right] } \\
& \times\left[\begin{array}{cc}
\boldsymbol{\Omega}\left(\Omega_{0}, P\right) & 0 \\
0 & m I_{n_{z}}
\end{array}\right] \\
& \times\left[\begin{array}{ccc}
A_{\mathrm{p}} & B_{\mathrm{p}} & \left.\begin{array}{c}
B_{\mathrm{a}, d} \\
0
\end{array}\right] \\
\gamma C_{\mathrm{p}} & 0 & 0
\end{array}\right] \\
& -\left[\begin{array}{ccc}
\boldsymbol{\Omega}\left(\Omega_{0}, P\right) & 0 & 0 \\
0 & m I_{n_{w}} & 0 \\
0 & 0 & \eta I_{m}
\end{array}\right]
\end{aligned}
$$

and where $B_{\mathrm{a}, d}$ is as in (23). For some sufficiently small scalar $m_{0}>0$, and for any $k \in \mathbb{N}^{+}$, we thus have that

$$
\begin{array}{r}
\left\|\left[e_{k}^{\mathrm{d}}, c_{k}^{\mathrm{d}}, K\left(\left[c_{k}^{\mathrm{d}}, \hat{d}_{k}\right]\right), w_{k}^{\mathrm{d}}, d_{k}\right]\right\|_{\boldsymbol{\Phi}\left(\Omega_{0}, P, m, \gamma, \eta\right)}^{2} \\
\quad \leq-m_{0}\left\|\left[e_{k}^{\mathrm{d}}, c_{k}^{\mathrm{d}}, v_{k}^{*}\right]\right\|^{2} .
\end{array}
$$

This inequality may be rearranged using (23), (60) and $v_{k}^{*}=$ $D_{1} K\left(\left[c_{k}^{\mathrm{d}}, \hat{d}_{k}\right]\right)$ to obtain

$$
\begin{aligned}
& \left\|\left[e_{k+1}^{\mathrm{d}}, c_{k+1}^{\mathrm{d}}, \Gamma K\left(\left[c_{k}^{\mathrm{d}}, \hat{d}_{k}\right]\right)\right]\right\|_{\boldsymbol{\Omega}\left(\Omega_{0}, P\right)}^{2}-V\left(e_{k}^{\mathrm{d}}, c_{k}^{\mathrm{d}}, \hat{d}_{k}\right) \\
& \leq-m_{0}\left\|\left[e_{k}^{\mathrm{d}}, c_{k}^{\mathrm{d}}, v_{k}^{*}\right]\right\|^{2}-m\left(\gamma^{2}\left\|z_{k}^{\mathrm{d}}\right\|^{2}-\left\|w_{k}^{\mathrm{d}}\right\|^{2}\right)+\eta\left\|d_{k}\right\|^{2} .
\end{aligned}
$$

Furthermore, since $\Gamma K\left(\left[c_{k}^{\mathrm{d}}, \hat{d}_{k}\right]\right)$ is a feasible solution to the optimization problem at time $k+1$ [see Theorem 3.1], a lower bound on the first term on the left hand side is

$$
\begin{aligned}
& \left\|\left[e_{k+1}^{\mathrm{d}}, c_{k+1}^{\mathrm{d}}, \Gamma K\left(\left[c_{k}^{\mathrm{d}}, \hat{d}_{k}\right]\right)\right]\right\|_{\boldsymbol{\Omega}\left(\Omega_{0}, P\right)}^{2} \\
& \quad=\left\|\left[e_{k+1}^{\mathrm{d}}, c_{k+1}^{\mathrm{d}}\right]\right\|_{\Omega_{0}}^{2}+\left\|\left[c_{k+1}^{\mathrm{d}}, \Gamma K\left(\left[c_{k}^{\mathrm{d}}, \hat{d}_{k}\right]\right)\right]\right\|_{P}^{2} \\
& \quad \geq V\left(e_{k+1}^{\mathrm{d}}, c_{k+1}^{\mathrm{d}}, \hat{d}_{k+1}\right) .
\end{aligned}
$$

Thus, we have established the "dissipation inequality"

$$
\begin{aligned}
& V\left(e_{k+1}^{\mathrm{d}}, c_{k+1}^{\mathrm{d}}, \hat{d}_{k+1}\right)-V\left(e_{k}^{\mathrm{d}}, c_{k}^{\mathrm{d}}, \hat{d}_{k}\right) \\
& \leq-m_{0}\left\|\left[e_{k}^{\mathrm{d}}, c_{k}^{\mathrm{d}}, v_{k}^{*}\right]\right\|^{2}-m\left(\gamma^{2}\left\|z_{k}^{\mathrm{d}}\right\|^{2}-\left\|w_{k}^{\mathrm{d}}\right\|^{2}\right)+\eta\left\|d_{k}\right\|^{2}
\end{aligned}
$$

for all $k \in \mathbb{N}^{+}$. Summing the above inequality over the interval $[0, L], L \in \mathbb{N}^{+}$, and using $V(\cdot) \geq 0$, leads to

$$
\begin{aligned}
& m_{0} \sum_{k=0}^{L}\left\|\left[e_{k}^{\mathrm{d}}, c_{k}^{\mathrm{d}}, v_{k}^{*}\right]\right\|^{2} \leq V\left(e_{0}^{\mathrm{d}}, c_{0}^{\mathrm{d}}, \hat{d}_{0}\right) \\
& \quad+m \sum_{k=0}^{L}\left(\left\|w_{k}^{\mathrm{d}}\right\|^{2}-\gamma^{2}\left\|z_{k}^{\mathrm{d}}\right\|^{2}\right)+\eta \sum_{k=0}^{L}\left\|d_{k}\right\|^{2} .
\end{aligned}
$$

Furthermore, use of Lemma B.2 yields

$m_{0} \sum_{k=0}^{L}\left\|\left[e_{k}^{\mathrm{d}}, c_{k}^{\mathrm{d}}, v_{k}^{*}\right]\right\|^{2} \leq V\left(e_{0}^{\mathrm{d}}, c_{0}^{\mathrm{d}}, \hat{d}_{0}\right)+m c+\eta \sum_{k=0}^{L}\left\|d_{k}\right\|^{2}$.

Since $m_{0}>0,\left\{d_{k}\right\} \in \ell_{2}$ and (109) holds for any $L \in \mathbb{N}^{+}$, it follows that $\left\{\left[e_{k}^{\mathrm{d}}, c_{k}^{\mathrm{d}}, v_{k}^{*}\right]\right\}_{k=0}^{\infty} \in \ell_{2}$, and since (from (75))

$$
\begin{aligned}
& x_{k}-\mathbf{x}^{*}(\Delta)=G_{1} e_{k}^{\mathrm{d}}+\left[\begin{array}{ll}
I & 0
\end{array}\right] c_{k}^{\mathrm{d}} \\
& u_{k}-\mathbf{u}^{*}(\Delta)=G_{2} e_{k}^{\mathrm{d}}+v_{k}^{*}
\end{aligned}
$$

this completes the proof.

\section{APPENDIX C \\ EXTENSION TO UNSTABLE SYSTEMS}

Here we generalize the results of Section III by considering a more general candidate policy, namely, $u_{k}=\hat{u}_{k}^{*}-F c_{k}^{\mathrm{d}}$, where $F$ is a suitably selected feedback gain. In closed-loop, we have

$$
\begin{aligned}
u_{k} & =\hat{u}_{k}^{*}-F c_{k}^{\mathrm{d}}+v_{k}^{*} \\
& =-\left[C(I-A)^{-1} B\right]^{-1} \hat{d}_{k}-F c_{k}^{\mathrm{d}}+v_{k}^{*}
\end{aligned}
$$

where $v_{k}^{*}$ is determined by the MPC policy (introduced below). Note that we recover (18) when $F=0$. By considering (112) we can address the case when $A$ is unstable, provided the following assumption holds:

Assumption C.1: The matrix $(I-A)$ is full rank, and the matrices $A_{L}$ [see (10)] and

$$
A_{0}(F) \triangleq\left[\begin{array}{cc}
A & 0 \\
0 & 0
\end{array}\right]-\left[\begin{array}{c}
B \\
I
\end{array}\right] F
$$

are stable.

In view of (112), we propose the following generalization of Algorithm 3.1 (however we keep the state- and steady-state target estimator of Section III): 
Algorithm C.1: At time $k \in \mathbb{N}^{+}$, given $u_{k-1}, y_{k}$ and $\left[\hat{x}_{k}, \hat{d}_{k}\right]$, compute (16), (21)

$$
\begin{aligned}
K\left(\left[c_{k}^{\mathrm{d}}, \hat{d}_{k}\right]\right) & \triangleq \arg \min _{\mu \in \mathbf{R}^{N n}}\left\{\left\|\left[c_{k}^{\mathrm{d}}, \mu\right]\right\|_{P}^{2} \text { s.t. }\left[c_{k}^{\mathrm{d}}, \mu\right] \in \mathbb{S}\left(\hat{d}_{k}\right)\right\} \\
u_{k} & =\hat{u}_{k}^{*}-F c_{k}^{\mathrm{d}}+D_{1} K\left(\left[c_{k}^{\mathrm{d}}, \hat{d}_{k}\right]\right)
\end{aligned}
$$

and update the estimator using (9). Then apply $u_{k}$ to the system (3). Here, the set $\mathbb{S}\left(\hat{d}_{k}\right)$ is as explained in Section III but using $A_{0} \leftarrow A_{0}(F)$ [see (113)] and $C_{q} \leftarrow C_{q}(F) \triangleq C_{q}-D_{q} F$ in (38)-(45).

By following the lines of Section III (but using Algorithm C.1 instead of Algorithm 3.1) we obtain the following generalized version of Theorem 3.3 (i.e., we recover Theorem 3.3 when $F=$ $0)$.

Theorem C.1 (Robust Stability Test): Let Assumption C.1, Co and (62) hold. Suppose that $\mathbf{C 1}$ and Condition 3.1 hold using

$$
\begin{aligned}
A_{\mathrm{a}} & \leftarrow A_{\mathrm{a}}(F) \triangleq\left[\begin{array}{cc}
A_{L} & 0 \\
\Lambda_{1} & A_{0}(F)
\end{array}\right] \\
C_{\mathrm{a}, z} & \leftarrow C_{\mathrm{a}, z}(F) \triangleq\left[\begin{array}{ll}
\Lambda_{3} & \left.\left(\left[\begin{array}{ll}
C_{z} & 0
\end{array}\right]-D F\right)\right]
\end{array}\right.
\end{aligned}
$$

in (23), and that $\mathbf{C 2}$ holds using $A_{0} \leftarrow A_{0}(F)$

$$
\begin{aligned}
\hat{A} \leftarrow \hat{A}(F) \triangleq & {\left[\begin{array}{cc}
A_{0} & B_{I} \\
0 & I
\end{array}\right] } \\
& -\left[\begin{array}{c}
B_{I} \\
0
\end{array}\right] F\left[I \quad\left[\begin{array}{c}
(I-A)^{-1} B \\
I
\end{array}\right]\right], \\
\hat{C} & \left.\leftarrow \hat{C}(F) \triangleq\left[\begin{array}{ll}
C_{q} & D_{q}
\end{array}\right]-D_{q} F\left[\begin{array}{cc}
I & (I-A)^{-1} B \\
I
\end{array}\right]\right], \\
K_{2} & \leftarrow K_{2}(F) \triangleq\left(C_{q}(F)+\left[\begin{array}{ll}
0 & D_{q}
\end{array}\right]\right) K_{1}
\end{aligned}
$$

in (30)-(36). Then Algorithm C.1 robustly stabilizes the system (3).

Proof: Using the substitutions described above the result may be proven in the same manner as Theorem 3.3. In particular, note that "natural" generalizations of Theorems 3.1 and 3.2 hold.

\section{REFERENCES}

[1] M. V. Kothare, V. Balakrishnan, and M. Morari, "Robust constrained model predictive control using linear matrix inequalities," Automatica, vol. 32, pp. 1361-1379, 1996.

[2] B.-G. Park, J.-W. Lee, and W. H. Kwon, "Robust one-step receding horizon control for constrained systems," Int. J. Robust Nonlin. Control, vol. 9, no. 7, pp. 381-395, 1999.

[3] B. Kouvaritakis, J. A. Rossiter, and J. Schuurmans, "Efficient robust predictive control," IEEE Trans. Autom. Control, vol. 45, no. 8, pp. 1545-1549, Jul. 2000.

[4] A. Casavola, D. Famularo, and G. Franzé, "Robust constrained predictive control of uncertain norm-bounded linear systems," Automatica, vol. 40, no. 11, pp. 1865-1876, 2004.

[5] B. Pluymers, J. A. Rossiter, J. A. K. Suykens, and B. De Moor, "A simple algorithm for robust MPC," in Proc. 16th IFAC World Congress, 2005, [CD ROM].

[6] L. Imsland, J. A. Rossiter, B. Pluymers, and J. Suykens, "Robust triple mode MPC," in Proc. Amer. Control Conf., Minneapolis, MN, 2006, pp. 869-874.

[7] M. Canale and M. Milanese, "Robust design of predictive controllers in presence of unmodeled dynamics," Eur. J. Control, vol. 9, no. 5, pp. 499-506, 2003.
[8] W. P. Heath, A. G. Wills, and J. A. G. Akkermans, "A sufficient condition for the stability of optimizing controllers with saturating actuators," Int. J. Robust Nonlin. Control, vol. 15, pp. 525-529, 2005.

[9] W. P. Heath and A. G. Wills, "The inherent robustness of constrained linear model predictive conrol," in Proc. 16th IFAC World Congress, 2005, [CD ROM].

[10] W. P. Heath, G. Li, A. G. Wills, and B. Lennox, "The robustness of input constrained model predictive control to infinity-norm bound model uncertainty," in Proc. 5th IFAC Symp. Robust Control Design, Toulouse, France, Jul. 2006, [CD ROM].

[11] G. Li, W. P. Heath, and B. Lennox, "The stability analysis of systems with nonlinear feedback expressed by a quadratic program," in Proc. 45th IEEE Conf. Decision Control, San Diego, CA, 2006, pp. $4247-4252$.

[12] C. Løvaas, M. M. Seron, and G. C. Goodwin, "Robust model predictive control of input-constrained stable systems with unstructured uncertainty," in Proc. Eur. Control Conf., Kos, Greece, 2007, pp. 3303-3310.

[13] C. Løvaas, "Dissipativity, Optimality and Robustness of Model Predictive Control Policies" Ph.D. dissertation, School Elect. Eng. Comp. Sci., Univ. Newcastle, , 2008 [Online]. Available: http://hdl.handle.net/ 1959.13/29571, doi

[14] C. Løvaas, M. M. Seron, and G. C. Goodwin, "Robust output-feedback model predictive control for systems with unstructured uncertainty," Automatica, vol. 44, no. 8, pp. 1933-1943, 2008.

[15] C. Løvaas, M. M. Seron, and G. C. Goodwin, "Robust output-feedback MPC with soft state constraints," in Proc. 17th IFAC World Congress, Seoul, Korea, 2008, pp. 13158-13162.

[16] Y. I. Lee and B. Kouvaritakis, "Receding horizon output feedback control for linear systems with input saturation," Proc. Inst. Elect. Eng., vol. 148, no. 2, pp. 109-115, 2001.

[17] L. Chisci, J. A. Rossiter, and G. Zappa, "Systems with persistent disturbances: Predictive control with restricted constraints," Automatica, vol. 37, no. 7, pp. 1019-1028, 2001.

[18] D. Q. Mayne, S. V. Raković, R. Findeisen, and F. Allgöwer, "Robust output feedback model predictive control of constrained linear systems," Automatica, vol. 42, no. 7, pp. 1217-1222, 2006.

[19] K. R. Muske and T. A. Badgwell, "Disturbance modeling for offset-free linear model predictive control," J. Process Control, vol. 12, no. 5, pp. 617-632, 2002.

[20] G. Pannocchia and J. B. Rawlings, "Disturbance models for offset-free model-predictive control," AIChE J., vol. 49, no. 2, pp. 426-437, 2003.

[21] D. Angeli, A. Casavola, and E. Mosca, "Predictive PI-control of linear plants under positional and incremental input saturations," Automatica, vol. 36, no. 10, pp. 1505-1516, 2000.

[22] G. Pannocchia, "Robust model predictive control with guaranteed setpoint tracking," J. Process Control, vol. 14, no. 8, pp. 927-937, 2004.

[23] G. Pannocchia and E. C. Kerrigan, "Offset-free receding horizon control of constrained linear systems," AIChE J., vol. 51, pp. 3134-3146, 2005.

[24] I. Alvarado, D. Limon, T. Alamo, and E. F. Camacho, "Output feedback robust tube based MPC for tracking of piece-wise constant references," in Proc. 46th IEEE Conf. Decision Control, New Orleans, LA, 2007, pp. 2175-2180.

[25] C. A. Desoer and M. Vidyasagar, Feedback Systems: Input-Output Properties. New York: Academic Press, 1975.

[26] G. Pannocchia and A. Bemporad, "Combined design of disturbance model and observer for offset-free model predictive control," IEEE Trans. Autom. Control, vol. 52, no. 6, pp. 1048-1053, Jun. 2007.

[27] A. Richards and J. P. How, "Robust stable model predictive control with constraint tightening," in Proc. Amer. Control Conf., Minneapolis, MN, 2006, pp. 1557-1562.

[28] I. Kolmanovsky and E. G. Gilbert, "Theory and computation of disturbance invariant sets for discrete-time linear systems," Math. Prob. Eng., vol. 4, no. 4, pp. 317-367, 1998.

[29] M. C. De Oliveira, J. C. Geromel, and J. Bernussou, "Extended $\mathrm{H}_{2}$ and $H_{\infty}$ norm characterizations and controller parametrizations for discrete-time systems," Int. J. Control, vol. 75, no. 9, pp. 666-679, 2002.

[30] S. Skogestad, M. Morari, and J. C. Doyle, "Robust control of ill-conditioned plants: High-purity distillation," IEEE Trans. Autom. Control, vol. AC-33, no. 12, pp. 1092-1105, 1988.

[31] S. V. Raković, E. C. Kerrigan, K. I. Kouramas, and Q. Mayne, "Invariant approximations of the minimal robust positively invariant set," IEEE Trans. Autom. Control, vol. 50, no. 3, pp. 406-410, Mar. 2005.

[32] P. O. M. Scokaert and J. B. Rawlings, "Feasibility issues in linear model predictive control," AIChE J., vol. 45, no. 8, pp. 1649-1659, 1999. 
[33] J. Löfberg, "YALMIP: A toolbox for modeling and optimization in MATLAB," in Proc. CACSD Conf., Taipei, Taiwan, 2004, pp. 284-289.

[34] J. F. Sturm, "Using SeDuMi 1.02, a MATLAB toolbox for optimization over symmetric cones," Optim. Methods Software, vol. 11-12, pp. 625-653, 1999.

[35] M. Kvasnica, P. Grieder, and M. Baotić, Multi-Parametric Toolbox (MPT) 2004 [Online]. Available: http://control.ee.ethz.ch/ mpt/

[36] E. C. Kerrigan, Matlab Invariant Set Toolbox 2001 [Online]. Available: www-control.eng.cam.ac.uk/eck21/matlab/invsetbox/

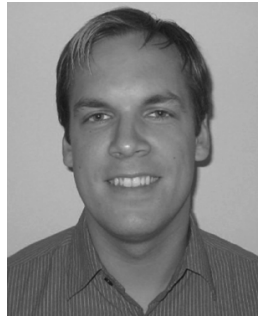

Christian Løvaas received the M.Sc. degree from the Norwegian University of Science and Technology, Trondheim, Norway, in 2004 and the Ph.D. degree from the University of Newcastle, Newcastle, Australia, in 2008.

During 2002/2003, he was a Visiting Postgraduate student at the University of New South Wales. In 2004, he received a full Ph.D. scholarship from the Research Council of Norway. He is currently with Statoil ASA, Kollsnes, Norway, where he works in the field of natural gas processing.

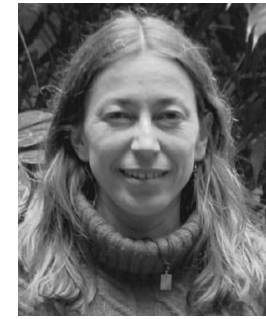

María M. Seron was born in Rosario, Argentina in 1963. She received the Electronic Engineer degree from the Universidad Nacional de Rosario, Argentina in 1988 and the Ph.D. degree from The University of Newcastle, Newcastle, Australia in 1996.

She held post-doctoral positions with the Centre for Systems Engineering and Applied Mechanics (CESAME), Catholic University of Louvain, Belgium, and with the Center for Control Engineering and Computation, University of California, Santa Barbara. From 1999 to 2002, she was an Associate Professor with the Department of Electronic Engineering, Universidad Nacional de Rosario, Argentina. She is currently an Australian Research Fellow with the Centre for Complex Dynamic Systems and Control, the University of Newcastle.

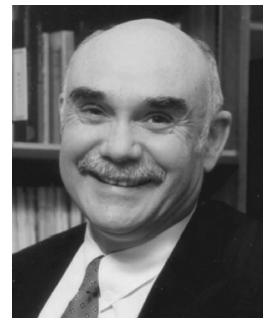

Graham C. Goodwin (M'74-SM'84-F'86) received the B.Sc degree in physics, the B.E degree in electrical engineering, and Ph.D. degree from the University of New South Wales, Sydney NSW 2052, Australia.

$\mathrm{He}$ is currently Professor of Electrical Engineering at the University of Newcastle, Newcastle, Australia. $\mathrm{He}$ is the co-author of eight books, four edited volumes, and many technical papers.

Dr. Goodwin received the Control Systems Society 1999 Hendrik Bode Lecture Prize, a Best Paper award from the IEEE TRANSACTIONS ON AUTOMATIC CONTROL, a Best Paper award from the Asian Journal of Control, and two Best Engineering Text Book awards from the International Federation of Automatic Control. $\mathrm{He}$ is an Honorary Fellow of the Institute of Engineers, Australia; a Fellow of the Australian Academy of Science; a Fellow of the Australian Academy of Technology, Science and Engineering; a Member of the International Statistical Institute; a Fellow of the Royal Society, London, and a Foreign Member of the Royal Swedish Academy of Sciences. He holds Honorary Doctorates from the Lund Institute of Technology, Lund, Sweden and the Technion, Haifa, Israel. 